\title{
Terahertz Emitters, Detectors and Sensors: Current Status and Future Prospects
}

\author{
M. Ghanashyam Krishna ${ }^{1,2}$, Sachin D. Kshirsagar ${ }^{1}$ and Surya P. Tewari1, \\ ${ }^{1}$ Advanced Centre of Research in High Energy Materials \\ ${ }^{2}$ School of Physics, University of Hyderabad, Hyderabad
}

India

\section{Introduction}

The region of the electromagnetic spectrum from 0.3 to $20 \mathrm{THz}(10-600 \mathrm{~cm}-1,1 \mathrm{~mm}-15 \mu \mathrm{m}$ wavelength) is an area for research that encompasses physics, chemistry, biology, materials science and medicine. The lack of sources of high quality radiation has limited developments in this area. However, there has been an upsurge of activity in the last five years due to the emergence of a wide range of new technologies. Terahertz radiation is now available in both continuous wave (cw) and pulsed form, down to single-cycles or less, with peak powers up to $10 \mathrm{MW}$ [Ginzburg et al., 2011; Sizov\& Rogalski, 2010; Williams, 2004; Tochitsky et al., 2005]. There is a wealth of information that can be extracted from a study of matter (solid, liquid or gaseous) at these frequencies. It is becoming evident that such directed basic research would also lead to a phenomenally large number of applications covering Physics, Chemistry, Life Sciences and Engineering [Jacobsen et al., 1996; Mueller, 2003; Chamberlain, 2004; Bolivar et al., 2004; Lewis, 2007; Tonouchi, 2007] .

As stated earlier, to achieve further understanding of physical phenomena in the $\mathrm{THz}$ region of the electromagnetic spectrum it is essential that there are reliable sources (including materials, devices and integrated systems) that emit $\mathrm{THz}$ radiation. Furthermore, there is need for materials, devices and systems that detect this radiation. Another aspect, which is central to all developments in $\mathrm{THz}$ science and technology is the development of reliable standards. Thus, THz metrology is another area that has to develop simultaneously with development of emitters and detectors. One of the applications, which is still in its infancy but has remarkable potential, is $\mathrm{THz}$ sensors for a variety of contexts in security, life sciences and medicine.

The aim of this review is to present the state-of-science and technology in the area of $\mathrm{THz}$ emitters, detectors and sensors. A simple search carried out on a general search engine with keywords "THz detectors or THz emitters" comes up with thousands of hits. Even a search on more focused science literature search engines and limited to the last five years, with the same keywords, generates over a thousand papers. This in essence, reveals the explosion of interest in the area of $\mathrm{THz}$ science as well as the ease of availability of sources, probes and tools for such studies. Therefore, the current review is limited to, what the authors consider, the most interesting and significant recent developments in the area of $\mathrm{THz}$ emitters, detectors and sensors. It is aimed at researchers intending to initiate work in these fields and 
therefore a considerable portion is focussed on basic principles. The rest of the review is organized in the following fashion: Section 2 will review some of the fundamental phenomena that occur in the THz region of the em spectrum; Section 3 will present a review on $\mathrm{THz}$ emitters; Section 4 is focused on $\mathrm{THz}$ detectors and sensors and; Section 5 will present an overview of the materials development work being carried out by the present authors. Section 6 will provide a summary and outlook for the future in this field

\section{Significance of $\mathrm{THz}$}

The $\mathrm{THz}$ spectral range is characterized by several unique features [Jacobsen et al., 1996; Mueller, 2003; Chamberlain, 2004; Bolivar et al., 2004; Lewis, 2007; . Innocenzi et al., 2009; Roeser et al. 2010]. It is the highest frequency band where the field can be measured coherently without an interferometer. The $\mathrm{THz}$ region of the spectrum is sensitive to all of the thermally accessible excitations that determine the properties of correlated electron systems. Also, the ability to perform spectroscopy in the time as well as frequency-domain provides a unique window on the temporal evolution of optical response functions on time scales between $100 \mathrm{fs}$ and 500 ps. Furthermore, THz methods are compatible with the study of materials under extreme conditions of temperature, electric, and magnetic field. $\mathrm{THz}$ probes are, in general, non-contact enabling polarization analysis and polarization control that may be of importance in situations where Ohmic contacts to certain materials are not possible due to the remote location of the material, or to avoid the introduction of extrinsic effects associated with leads. Terahertz probes can also provide detailed information on interface quality or on the presence of buried defects or structures.

Two, one and zero dimensionally quantum confined systems have electronic excitations in the $\mathrm{THz}$ regime. As the $\mathrm{THz}$ frequency exceeds various relaxation rates and broadening, it is possible to achieve well defined collective and single particle excitations. As a consequence many fundamental issues relating to control of carrier injection by doping, the relationship between geometry and energy level structure, line widths of transitions between states, excited state coherence and population lifetimes, superposition states of carriers, and spin excitations can be probed. These are issues not only of interest from a basic perspective but also critical for applications such as sources, detectors, novel ultrafast electro-optic devices and quantum information processing in semiconductors [Tonouchi, 2007].

Another area of interest is the coherent quantum control of the orbital and spin states of carriers in semiconductor nanostructures that occur at $\mathrm{THz}$ frequencies. The properties of semiconductor nanostructures at visible and near-IR wavelengths can also be controlled with $\mathrm{THz}$-frequency electromagnetic radiation. In these structures, energy-level splittings are often in the $\mathrm{THz}$ range. At $\mathrm{THz}$ frequencies, electron interactions with acoustic and optical phonons are small leading to narrow line widths of electronic excitations. These small line widths and correspondingly long relaxation and dephasing times find application in a variety of devices, including light emitters, detector and quantum logic devices Magnetic level splittings in semiconductor nanostructures in magnetic fields of a few Tesla are frequently in the $\mathrm{THz}$ regime. Metals are typically very good reflectors of $\mathrm{THz}$ radiation. Majority of solids have optical phonons at $\mathrm{THz}$ frequencies. Most of these are infraredactive, which can be excited directly by resonant oscillating electric fields[Lewis, 2007]. Phonon features are particularly interesting near structural phase transitions, where they become "soft" and move towards zero frequency. Phonons interact strongly with electrons, 
a phenomenon that was central to explaining conventional superconductivity. A Josephson plasma resonance is produced by coherent propagation of Cooper pairs between superconducting "sheets" in layered superconductors. In a variety of layered superconductors this mode falls in THz frequency range[ Tonouchi, 2007; Ferguson \& Zhang, 2002; Sizov \& Rogalski, 2010] Other similar strongly correlated electron systems have very complex phase diagrams with several transitions among them. Understanding the nature of these phases, as well as the transitions among them, requires detailed characterization of their excited states, or elementary excitations. The most important excitations are those with an energy of order $k_{B} T$ above the ground state and therefore in the $\mathrm{THz}$ region of the electromagnetic spectrum. Fundamental interactions between quasiparticles, phonons, spin-excitations and other constituents of correlated materials occur on ultrafast time scales [ Kida et al., 2005]. It is worth noting that the phonon modes of inorganic and organic crystals fall in the THz region of the spectrum [Zheng et al., 2007].

The classical rotation period for a nitrogen molecule with one $\hbar$ of angular momentum is about a pico second. Similarly, the time of a molecular collision at room temperature is 0.1 to 1 ps. At very low temperatures the collisional energy of molecular collisions is comparable to the rotational energy level spacing. As a consequence, the collisions are quantum mechanical in nature and resonances in their cross sections occur in the $\mathrm{THz}$ region. The time scale of gas phase collisions is of the order $10^{-12}$ seconds which is ideally suited for $\mathrm{THz}$ studies. Rydberg atoms in the $n=20$ to $n=60$ range span the spectrum of sensitive response to $\mathrm{THz}$ fields. These are model systems for studying many aspects of few body quantum mechanics, including quantum chaos, quantum-classical correspondence, and quantum systems in the presence of strong external fields [Rangan \& Bucksbaum, 2001] .

Interaction of high-intensity femtosecond lasers with solid matter generates plasmas that can be employed as sources of short $x$-ray pulses, coherent harmonic radiation, energetic electron and ion beams, plasma-based accelerators, ultra high magnetic fields. These plasmas can also serve as a source of intense THz emission [Li et al, 2011].

There are several processes in liquids that occur in the $\mathrm{THz}$ region and lend themselves to be probed using $\mathrm{THz}$ radiation. $\mathrm{THz}$ spectroscopy can also reveal information about protein structure and dynamics. It is possible to distinguish many amino acids based on their $\mathrm{THz}$ spectrum, particularly in the crystalline form. The THz spectra of individual DNA base pairs can be used to understand dynamics and conductivity. It could lead to label-free measurement of protein-protein interactions as cellular activity is occurring in live cells. $\mathrm{THz}$ radiation holds promise in new medical imaging techniques based on pulsed and $\mathrm{cw}$ $\mathrm{THz}$ sources. Differences in tissue water, architecture and chemical content can easily be detected using $\mathrm{THz}$ techniques since this radiation is strongly absorbed by water. This can lead to early detection of diseases by revealing features that are not apparent with other imaging techniques. [Nagel etal., 2003] .

It is evident that $\mathrm{THz}$ region is a region of the electromagnetic spectrum that is rich with information on all forms of matter. It is further obvious that significant developments in this area are technology limited rather than being limited by science.

The research and development in $\mathrm{THz}$ science and technology can be broadly divided in to the following categories based on the fundamental phenomena that occur at the $\mathrm{THz}$ region 
of the em spectrum. In all these cases there are issues relating to materials, processing technologies, devices, and system integration issues need to be addressed.

1. Sources

2. Emitters

3. Detectors and sensors

It is pertinent at this point to distinguish between detectors and sensors. For purposes of this review, detectors are used to signify components that provide only frequency information of the $\mathrm{THz}$ radiation. Sensors, in contrast, provide information on both the frequency and time domain as well as the source that causes variation in frequency of radiation incident on the detector. Detectors, thus, form a sub-set of the sensor. Sensors can, therefore be used for imaging, spectroscopy and sensing the nature of environment around the $\mathrm{THz}$ emitter or detector.

\section{THz emitters}

Terahertz radiation has potential in various short-wavelength communication devices and security applications. In order to attain the potential offered by terahertz technology, design of solid state terahertz emitters is indispensible. Terahertz sources based on femto second lasers pulses is one of the most promising techniques to generate $\mathrm{THz}$ radiation. Lasers are strong sources of electromagnetic energy where energy is stored both in the intensity and frequency. THz generation by ultrafast laser pulses can be classified as linear and non linear optical processes. The linear process involves injection of photocarriers by a laser pulse into the semiconductor connected to the antenna. The photocarriers are accelerated by dc bias applied to the antenna. The antenna radiates $\mathrm{THz}$ signal of a frequency that is determined by the pulse duration of laser pulses. In contrast, in the nonlinear optical process $\mathrm{THz}$ radiation is generated by optical rectification or difference frequency mixing techniques. The nonlinear optics methods are attractive for terahertz devices because of several properties, including (i) possibility of room-temperature operation (ii) easy operation. However, one of the major hurdles in operating terahertz emitters lies in poor power conversion efficiency, which is of the order of $10^{-6}$ to $10^{-5}$. Terahertz sources can be divided according to their bandwidth, output power, and operating principle [Sakai \& Tani, 2005]. In following sections we focus on the several kinds of terahertz emitters. Brief theories of the operating principle have been put forth with the explanation of their merits and demerits.

\subsection{Photoconductive broadband THz antenna}

An ultrashort laser pulse incident on the surface of a photoconductor at an angle of incidence $\theta$, generates photocarriers, which then move under the influence of an electrical bias field. The bias field may be either externally applied (parallel or perpendicular to the surface) or internally generated by band bending from trapped surface charges (perpendicular to the surface). The resulting transient photocurrent gives rise to the emission of $\mathrm{THz}$ electromagnetic pulses in the reflected and transmitted directions. When a femto second (fs) laser pulse, with an arbitrary intensity profile, excites a biased semiconductor with photon energy greater than its bandgap, electrons and holes are produced at the illumination point in conduction and valence bands, respectively. The rapid changes of the density of photocarriers and their acceleration due to the applied DC bias 
produce an electromagnetic field radiation into free-space with the help of an antenna. The production of ultrashort currents with a FWHM lifetime of 1 ps or less strongly depends on the carrier's lifetime in the semiconductor. The space-charge screening and radiation-field screening are two factors preventing photoconductor from generating higher power $\mathrm{THz}$ radiation, which could affect all kinds of the photoconductive antenna. Based on the shape of antenna, their effects are different. Generally speaking, the space-charge screening can be important to small dipole antennas, but, for large area antennas, radiation-field screening is the major cause of saturation. Photoconducting switches are commonly used in timedomain terahertz spectroscopy, where, a generation and detection $\mathrm{THz}$ wave is carried out by use of biased photoconducting GaAs aperture antenna. The advantage of semiconductor photoconductive switches is that they can be used to efficiently generate signals and to generate and detect electrical transients in guided media or free space. However, the problem with photoconductive switches is that electrical pulse width is limited by a number of factors such as the laser pulse width, circuit parameters of the generation and detection site, and the carrier lifetime in the semiconductor. Furthermore, the carriers can be captured by traps at the grain boundaries, resulting in a rapid decay in number of carriers [Sizov \& Rogalski, 2010].

Usually materials with reduced carrier lifetimes and high carrier mobility such as LT-GaAs or ion implanted semiconducting layers are utilized. Ga doped InAs based photo conductive switches are expected to yield higher terahertz output because of their higher electron mobility [Lloyd-Hughes et al., 2005]. Liu et al. report improvement in photoconductive response in shorter terahertz emission pulses from spiral antennas fabricated on multienergy-implanted GaAs:As[Liu et al., 2005]. This is due to the uniformity of arsenic antisite defects (traps) distributed in multi-energy-implanted GaAs:As which contributes to the unchanged carrier trapping time as compared to single-energy-implanted GaAs:As. Miyadera et al. [Miyadera et al., 2004] used amorphous Ge thin films fabricated on $\left(\begin{array}{ll}1 & 0\end{array}\right) \mathrm{MgO}$ substrates as a terahertz emitter by coupling with $\mathrm{Au} / \mathrm{Cr}$ transmission lines. Amorphous $\mathrm{Ge}$ will be beneficial for optical to electrical signal converter, due to the potential advantages of fabrication without heating process and smaller bandgap of about $0.66 \mathrm{eV}$.

Light absorption process in the photoconductive switches which generates free carriers near the surface must also be considered. The absorption of terahertz pulses by free carrier can degrade overall efficiency of photoconductors. GaAs constituents of photoconductive

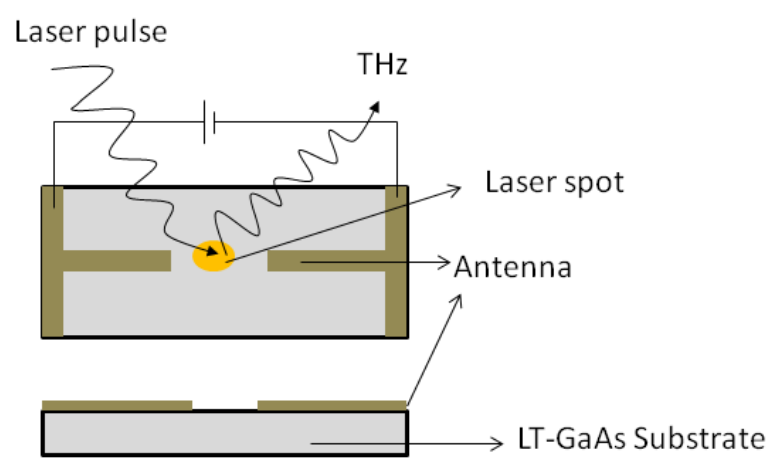

Fig. 1. Typical schematic of a photoconductive switch 
switches have a phonon resonance around $8 \mathrm{THz}$ that generates a broad absorption band between 7 and $10 \mathrm{THz}$. In order to reduce absorption caused by phonon resonance, Kasai et al. have fabricated LT-GaAs epitaxial layer which is transferred onto high resistivity Si substrates [Kasai et al., 2009]. These detectors showed higher sensitivity than conventional photoconducting switches at high frequencies. The typical schematic of a photoconductive switch is shown in fig 1.

\subsubsection{Photo-Dember effect emitter}

If surface energy bands of a semiconductor lie within its bulk band gap, then Fermi level pinning occurs, leading to band bending and the formation of a depletion/accumulation region at the surface. The resulting electric field will separate photo generated electrons and holes, forming a dipole perpendicular to the surface which emits $\mathrm{THz}$ radiation, this is the surface-field emission. By changing doping from $n$ type to $p$ type, the sign of the electricfield direction is reversed. This leads to a change in the polarity of the dipole, which is observed as a change in sign of the generated $\mathrm{THz}$ transient. A photo-Dember field can occur at the surface of a semiconductor after photo excitation. Two factors lead to this field: a difference in diffusion coefficients for electrons and holes, and a structural asymmetry. In a typical semiconductor, electrons have a larger diffusion coefficient than holes. Therefore, after photo excitation, the electron population diffuses more rapidly than the hole population. In the absence of a surface boundary, there would be no net dipole field, since the center of charge does not change. However, in the vicinity of the surface, reflection or capture of charges results in the center of charge for electrons and holes moving away from the surface. A dipole is thus formed perpendicular to the surface, leading to $\mathrm{THz}$ emission. In this case changing the semiconductor doping from $n$ to $p$ type has no effect on the sign of the emitted $\mathrm{THz}$ pulse. One of the main applications of photo-Dember effect is the generation of terahertz $(\mathrm{THz})$ radiation pulses for terahertz time-domain spectroscopy. This effect is present in most semiconductors but it is particularly strong in narrow-gap semiconductors (mainly arsenides and antimonides) such as InAs and InSb owing to their high electron mobility.[ Yi et al, 2010; Reklaitis, 2011; Klatt et al., 2010; Krotkus, 2010]

\subsubsection{Current transient effect emitter}

The current transient emitter or Auston switch is simply a gap in a uniform transmission line defined by thin-film metal fabrication on the top surface of an ultrafast photoconductor The transmission line is, generally, a coupled-strip line terminated in a planar antenna, within a planar strip dipole. The entire transmission line structure is biased with a voltage through an inductor. The principle of operation is that the gap in the transmission line initially creates an open-circuit condition. When a short pulse from a mode-locked laser arrives in the gap, a shower of electron-hole pairs are generated in the first few microns below the surface. The instantaneous concentration of these pairs is high enough to "shortout" the gap. When the laser pulse is terminated, the electron-hole pairs quickly recombine, creating an electrical impulse (or surge current) on the same time scale as the laser pulse. Due to the wide bandwidth of the planar transmission line, the electrical impulse propagates down the line to the antenna with minimal dispersion or droop. So upon reaching the antenna, the electrical pulse generates a significant amount of $\mathrm{THz}$ radiation that propagates primarily into the substrate.[Reimann, 2007; Suen et al., 2010;] 


\subsection{Optical rectification based emitters}

One of the more popular means of generating $\mathrm{THz}$ frequency radiation is through the bulk second-order nonlinear effect of optical rectification. Optical rectification occurs if highintensity light is directed onto an electro-optic material. If the excitation beam contains components of two or more frequencies then difference-frequency mixing, also known as optical rectification, may take place. Depending on the spectrum of the excitation beam, the resulting frequency may be in the $\mathrm{THz}$ range. The optical rectification process has been widely developed for $\mathrm{THz}$ generation, typically in materials with a completely nonresonant response. The advantage of such an approach is the inherent speed of the response, which may be viewed as essentially instantaneous. The disadvantage is the relative weakness of the nonlinear response. Thus, in order to obtain significant $\mathrm{THz}$ emission via the optical rectification mechanism it is desirable to use a medium of appreciable thickness which leads to the necessity of considering phase-matching constraints. The problem of broadband phase matching is a challenge. One possibility is exploiting the non-resonant second-order nonlinear response expected in any non-centrosymmetric semiconductor, such as the III-V materials. This effect can be identified by the distinctive dependence that it shows on the crystallographic orientation of the sample. Optical rectification using an ultra-short femto laser in the periodically poled structure can be considered as a special case of difference frequency mixing where terahertz radiation emitted from the polarization change that follows the transport of excited carriers in an applied or surface electric field. The physics of optical rectification leading to $\mathrm{THz}$ generation is well understood [Blanchard et. al, 2011]. The nonlinear coefficient of the second order susceptibility is an extremely important material property when high-power output $\mathrm{THz}$ emitters are considered. The output power is also predominantly determined by intensity of the excitation pulse and the phase matching conditions. The major disadvantage of this technique is low power output, broad line-width, velocity mismatch and requirement of expensive femto second laser sources. In addition, there are the restrictions implied by other nonlinear processes like two-photon absorption, competing with optical rectification and limiting its efficiency. The process of two-photon absorption not only influences efficiency of terahertz generation but also impacts the terahertz spectrum [Vidal et al., 2011]. THz generation via optical rectification of $\mathrm{x}$-mode laser in a rippled density magnetized plasma has been demonstrated [Bhasin and Tripathi [Bhasin \& Tripathi, 2009]. Fiber lasers are attractive as terahertz emitters because of their low cost, small size, high repetition rate, high pulse energy, and short pulse duration[Hoffmann et al., 2008].

Relativistic optical rectification, has opened up the potential realization of high frequency range terahertz emitter[Tsaur \& Wang, 2009]. It is should be noted that bandwidth of emitter is sharply reduced in optical rectification due to the phase-matching condition. For EO sampling and optical rectification, the spectral response of the material is strongly sensitive to reststrahlen band absorption/reflection, phase matching, dispersion of refractive index and dispersion of EO coefficients, which can be expressed as

$$
G(\Omega)=\frac{t(\Omega)(\exp (i 2 \pi \Omega \delta(\Omega))-1)}{\left[1-r(\Omega)^{2} \exp \left(\frac{i 4 \pi \Omega n(\Omega) d}{c}\right)(i 2 \pi \Omega \delta(\Omega))\right]}
$$




$$
\delta(\Omega)=\frac{n_{g}(\lambda)-n(\Omega)}{c} d
$$

$\delta(\Omega)$ is the velocity mismatch in time, $n_{g}(\lambda)$, is the optical group index, $n(\Omega)$, is the terahertz refractive index, $d$ is crystal thickness, $t(\Omega)$ and $r(\Omega)$ are the Fresnel transmission and reflection coefficients and the multi-reflection of terahertz field inside the sensor material. The velocity mismatch between phase velocity of terahertz pulse and group velocity of laser beam hampers output power of these sources. This could be eliminated by placing nonlinear crystal in hollow metallic waveguide structure [Nikoghosyan, 2010]. The conversion efficiency in optical rectification depends primarily on the material nonlinear coefficient and the phase-matching conditions and various schemes for power enhancement have been reported [Radhanpura et al., 2010; Bugay \& Sazonov, 2010; He et al., 2008; Reid et al., 2008]. To overcome the problem of phase mismatch, interaction of pump optical pulse with a twolevel resonant impurities, for the terahertz wave is proposed [Bugay \& Sazonov, 2010]. Alternatively, phase mismatching can also be effectively used to tune the operating frequency from 4.2 to $1.1 \mathrm{THz}$ of a (110) oriented ZnTe crystal terahertz emitter. Materials with large drift velocity and short carrier lifetime such as GaAs and ZnTe are popularly used in optical rectification techniques. However, they are limited by large band gap which hampers their efficiency [Li \& Ma, 2008]. In combination with second order susceptibility, effective second-order processes such as electric field induced optical rectification (EFIOR) has been shown to yield terahertz generation in centrosymmetric Ge crystals [Urbanowicz et al., 2007]. This effect has been termed EFIOR, because it is easily explained in the framework of third-order nonlinearity $\left(\chi^{(3)}\right)$ contributed to effective second order process by DC surface electric field.

\subsection{Quantum cascade laser emitters}

Energy levels in semiconductor quantum wells can be designed and engineered to be of any value, with ease. In such systems, inter sub band transitions involving electrons that make lasing transitions between subband levels within the conduction band can be exploited to realize emissions in the far infrared and $\mathrm{THz}$ regions. Such lasers are called quantum cascade lasers (QCLs). Simple calculations show that energy level separation to achieve emission in the $\mathrm{THz}$ region from QCLs is in the $\mathrm{meV}$ region $(1 \mathrm{THz}=4 \mathrm{meV})$. Design of quantum well structures for selective injection to the upper level and selective depopulation of electrons from the lower level is a challenge since, due to the narrow separation between subband levels, heating and electron-electron scattering have a great effect. Some of the other challenges that need to be overcome include detection and analysis of spontaneous emission; mode confinement at longer wavelengths; dielectric waveguide confinement (as the evanescent field penetration, which is proportional to the wavelength is of the order of several tens of microns $>>$ the active gain medium of several microns. The physical origin of the $\mathrm{THz}$ emission of the quantum cascade laser, as stated above, can be traced to intersubband transitions. The quantum well thickness in QCL is one of the key parameters to control in order to finely tune laser energy. The background blackbody radiation and/or emissions from impurity levels may lead to poor reproducibility of the results. The microprobe photoluminescence has been shown to provide a powerful tool for this purpose which can probe emission from individual states in the active region with the high spatial resolution [Freeman et al., 2011]. 
Unfortunately, it has been observed that QCL are not popular for $\mathrm{THz}$ source due to low energy (long wavelength) output. This is a consequence of the poor coupling between the small gain medium and the optical field. In fact, high optical losses are caused due to free electrons in the material at low energy. Recent developments in QCL technology have raised the hope for their application in terahertz devices. The nonequilibrium Green's function calculations performed by Yasuda et al. [Yasuda et al., 2009] predict that the 4L QCL has a larger terahertz gain than the conventional resonant phonon QCL. They have attributed it to the large number of electrons accumulate in the upper lasing level and contribute to lasing in the new scheme. They have found that the advantage of gain deteriorates at $200 \mathrm{~K}$ due to thermally activated phonon scattering.

Another major limitation of QCL is their low operation temperature. The maximum operating temperature of $178 \mathrm{~K}$ is currently the highest operating temperature achieved in a THz QCL. The need to use cryogenic cooling is considered to be a major obstacle to the introduction of room temperature QCL in terahertz devices. Ambient temperature operation can be achieved by integrating the optical nonlinearity for difference-frequency generation(DFG) into the active region of a dual-wavelength mid-IR QCL using band structure engineering of the QCL active region [Belkin et al., 2009]. However, the disadvantage of DFG QCLs is that they provide less terahertz power than normal QCL. Alternatively, it is also possible to improve temperature performance of QCL by implementation of a second type of waveguide (metal-metal (MM)) design which consists of metal films on both sides of the active region, and provides a mode confinement factor of nearly 100\% [Belkin et al., 2009]. Optimized Second Harmonic Generation (SHG) in QC lasers, in which the QWs of the active regions simultaneously function as nonlinear oscillators, have led to high absolute SHG power levels of up to $2 \mu \mathrm{W}$ and large linear-tononlinear power conversion efficiency of around 50-100 $\mu \mathrm{W} / \mathrm{W}^{2}[\mathrm{Gmachl}$ et al., 2003].

The present trend in the search of materials for QCL is to use silicon, which can offer the prospect of integrating coherent terahertz $(\mathrm{THz})$ radiation sources with silicon microelectronics. Silicon is the only material that allows mature processing technology which may reduce costs and allow integration with conventional electronic devices. A recent report on silicon based QCL focused on a variety of $n$-type SiGe-based heterostructures as design candidates [Valavanis et al., 2011]. They have carried out theoretical studies on (001) $\mathrm{Ge} / \mathrm{GeSi}$, (111) Si/SiGe, and (001) Si/SiGe material configurations where they have found that $(001) \mathrm{Ge} / \mathrm{GeSi}$ is the most promising system for development of a Si-based QCL

\subsection{Photomixers}

Modulated infrared radiation can cause the resonant excitation of plasma oscillations in quantum well diode and transistor structures with high electron mobility. This effect provides a mechanism for the generation of tunable terahertz radiation using photomixing of infrared signals [Preu et al., 2011] .

In terahertz photomixer, the outputs of two continuous-wave sources with frequency difference falling in the terahertz range mix in a photoconductors. The laser induced photocarriers short the gap of device producing photocurrent which is modulated at the laser difference frequency. The generated signal is usually fed to the antenna to transmit into free space. The photomixers are tuneable, coherent, compact, low cost and narrow-band 
$\mathrm{THz}$ radiation sources. Photomixers have the greatest tuning range among all the coherent sources in terahertz region. Note that photomixing is fundamentally different from difference frequency mixing. Photomixing is more efficient than difference frequency generation at low $\mathrm{THz}$ frequencies. At higher $\mathrm{THz}$ frequencies, nonlinearities are more efficient due to parasitic impedances which limit the $\mathrm{THz}$ bandwidth of photomixers [Mittleman, 2003]. In difference frequency mixing, the outputs of two laser sources are mixed in nonlinear medium like $\mathrm{ZnTe}$ or $\mathrm{LiNbO}_{3}$, where, an intense laser field can cause the polarization of the nonlinear medium to develop new frequency components. These resultant frequency components of polarization act as source of terahertz radiations.

The frequency and power of the generated terahertz signal can be tuned by tuning the central frequency of one of the lasers. Based on model calculations for LT-GaAs photomixer, it has been proposed [Pilla, 2007] that photomixing efficiency can be enhanced by reducing the transit time of majority of carriers in photomixers and photodetectors to $<1 \mathrm{ps}$. The model indicated that the output terahertz power, $\mathrm{P}_{\mathrm{f}}$ is proportional to difference frequency of incident lasers $(f)$ which is given by relation Pf a $\mathrm{f}^{-2.77}$ in the $0.5-6.5 \mathrm{THz}$ range.

In difference frequency mixing, an intense pulsed laser induces a significant polarization in non-centrosymmetric structured materials, which can be written as

$$
P=\chi^{(1)} E+\chi^{(2)} E
$$

where first term on the right hand side represents linear polarization while second term is responsible for SHG (second harmonic generation), dc rectification, Pockels effect, parametric generation etc

In nonlinear crystals, sum frequency generation (SFG) or difference frequency generation (DFG) can occur, where two pump beams generate another beam with the sum or difference of the optical frequencies of the pump beams. If the incident electric field contains two different frequency components $\omega_{\mathrm{p}}$ and $\omega_{\mathrm{s}}$ and amplitudes $\mathrm{E}_{\mathrm{g}}$ and $\mathrm{E}_{\mathrm{p}}$ respectively, the resultant intensity can written as

$$
2 \chi^{(2)} E_{p} E_{s}\left\{\cos \left(\omega_{p}+\omega_{s}\right) t+\cos \left(\omega_{p}-\omega_{s}\right) t\right\}
$$

It is clearly seen from equation (4) that the second order nonlinear susceptibility will give rise to a nonlinear polarization and reemit radiation at sum $\left(\omega_{p}+\omega_{s}\right)$ and difference $\left(\omega_{p}-\omega_{s}\right)$ frequencies.

Advantages associated with a difference frequency generation include simple frequency control, narrow linewidth and wide tuning ranges based on low loss and phase matched NLO crystals and tunable pump lasers. Diode lasers are sometimes used in difference frequency generation sources for large band width $\mathrm{THz}$ signals. Narrow-band terahertz pulse generation via difference frequency mixing using fs laser irradiation of periodically poled lithium niobate (PPLN) and stoichiometric lithium tantalate (PPSLT) crystals[Yu et al., 2011]. The bandwidth of output terahertz radiation obtained was as low as $32 \mathrm{GHz}$ for 1.38 and $0.65 \mathrm{THz}$ for forward and backward $\mathrm{THz}$ pulses respectively. The phase mismatch is another critical factor affecting performance of difference frequency generation sources. The conversion efficiency in difference frequency generation process depends primarily on the material's nonlinear coefficient and the phase (velocity) matching conditions. The wave 
vector of the applied field and the generated field must have the same relation as their frequencies. Unfortunately in nonlinear crystals, the optical beam always travels faster than the $\mathrm{THz}$ beam, which makes the phase matching unachievable. Several phase matching techniques been explored to improve output power of terahertz emitters. The phase matching techniques can reduce decay of terahertz radiation in the crystals by techniques like reducing beam diameter [Shibuya et al., 2010] or artificially introducing reversed domain structures (quasi-phase matching, QPM) [Marandi et al., 2008]

\section{THz detectors and sensors}

\subsection{Detectors}

$\mathrm{THz}$ detectors have been developed based on a variety of principles as described below. However there are two generalized performance parameters that characterize $\mathrm{THz}$ detectors. These are Noise Equivalent Power (NEP) and detectivity.

Noise Equivalent Power (NEP) is defined the incident RMS optical power required in order to obtain signal-to-noise ratio of 1 in a bandwidth of $1 \mathrm{~Hz}$. NEP is related to the minimum power that the photodetector can detect

$$
\mathrm{NEP}=\left\langle\mathrm{i}_{\mathrm{n}}{ }^{2}\right\rangle / \mathrm{R}
$$

Where $i_{\mathrm{n}}$ is the noise current, and $R$ is the detector responsivity. The related figure of merit is detectivity defined as

$$
\mathrm{D}^{*}=\sqrt{ } \mathrm{SB} / \mathrm{NEP}
$$

where B is the bandwidth and S is the detector area. [Sizov \& Rogalski, 2010]

\subsection{Direct and heterodyne THz detection based on the principle of bolometer}

Hot electron bolometers are very commonly used for $\mathrm{THz}$ detection. The term hot electron describes electrons which are not in thermal equilibrium with the lattice. In metals the heating of electrons does not change the electron mobility and therefore it does not affect the resistance value. The hot electron approach is very productive for semiconductors, where the mobility of electrons depends on their effective temperature. In a superconductor, the hot-electron phenomenon consists of the heating of electrons by radiation. A photon incident on a superconductor is absorbed by a Cooper pair. Due to the large coherence length only one of the electrons of the Cooper pair absorbs the photon. The Cooper pair breaks apart and one highly excited electron with energy close to the incident photon energy and one low-energy quasiparticle are created. Next the quasiparticle loses its energy via electron-electron scattering and creates a secondary excited electron. This process continues so long as the incident radiation is switched on. There are two different techniques to detect the power of submillimeter and far-infrared wavelengths. The first approach is to detect the radiation directly by creating charged carriers or converting the incoming energy to heat proportional to the flux of the incoming photons. The first method is direct detection which is also called an incoherent detection. Direct detectors do not have a fundamental limit of the sensitivity. A direct detector does not produce any noise power unless photons are absorbed. The second method is to shift the incoming radiation to a lower frequency band and then amplify and detect the power of the radiation. The method of shifting the 
incoming radiation to a lower frequency is called heterodyne principle or coherent detection. In a heterodyne receiver a locally generated frequency is mixed with a signal frequency to produce a signal at a much lower frequency. This frequency conversion is done by a mixer element. The locally generated frequency and the signal frequency are added together to produce a beat frequency, the difference between the signal and the locally generated frequency. The HEB can be used as a heterodyne mixer. The incident radiation and the local oscillator $(\mathrm{LO})$ radiation excite a voltage across the bolometer. The dissipated power of the bolometer is a function of the average absorbed signal power and the average absorbed LO power. The output power consists of a component equal to the difference frequency of the electronic diode. Thus, a HEB can be used as a heterodyne mixer, if it is fast enough to follow the IF [Cherednichenko et al., 2002, Zmuidzinas \& Richardds, 2004] .

Superconductor-insulator-superconductor (SIS) tunnel junctions are extremely sensitive heterodyne mixers and have been widely used for frequencies below $1 \mathrm{THz}$. The upper frequency limit of these devices is determined by the gap frequency of the superconductor defined by fgap $=\Delta / \mathrm{h}$ with $\Delta$ the superconducting gap energy and $\mathrm{h}$ the Planck's constant. Drastic losses above the gap frequency of the superconductor (about $700 \mathrm{GHz}$ for niobium) occur inside the superconducting tuning circuit which is used to match the impedance of the SIS device to the antenna impedance. Using superconductors (like $\mathrm{NbN}$ or $\mathrm{NbTiN}$ ) having a higher gap frequency than $\mathrm{Nb}$ has increased the upper frequency limit of SISmixers to about $1.2 \mathrm{THz}$. The main advantage of SIS mixers is the wide Intermediate Frequency (IF) bandwidth provided by this type of mixer. HEB mixers do not have an upper frequency limit and provide a very high sensitivity $\left(\mathrm{T}_{\text {rec }}<2000 \mathrm{~K}\right)$ and require very low LO power $(<1$ $\mu W)$. Although the IF bandwidth of HEB mixers is rather limited compared to Schottky and SIS mixers, HEB mixers are the most competitive devices for heterodyne detection in the $\mathrm{THz}$ range. When compared by characteristics like response time and dynamic range, SIS detectors are found to be much superior to their TES counterparts [Matsuo, 2006].

The use of low temperatures based on $\mathrm{Nb}, \mathrm{NbN}$ and a number of related compounds have been reported. In these cases while the fabrication of the bolometer structures is routine since feature sizes are large, the very low operating temperatures $(<20 \mathrm{~K}$, in some cases) is a major drawback [Chen et al., 2009; Tretyakov et al. ; Stern et al., 2005; Schultz \& Lichtenberger, 2007; Ilin et al., 2010; Liu et al., 2009; Kleinschmidt et al., 2007; Cherednichenko et al., 2007 ] . In addition, these bolometers work in the range $<5 \mathrm{THz}$, generally. The typical schematic view of a HEB structure is shown in figure 2.

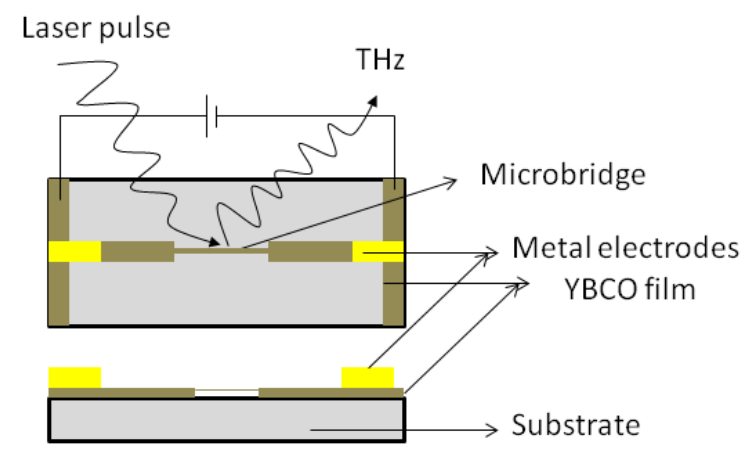

Fig. 2. The typical schematic view of a HEB structure 
Bolometers have also shown potential for application in space and astronomy [Meledin et al., 2004; Caumes et al., 2009; Putz et al., 2011]. The discovery of high temperature superconductors has helped in increasing the operating temperature of the HEBs to liquid nitrogen temperatures. For example, an ultrathin PBCO/YBCO/PBCO HEB [Peroz et al., 2007] constructed by ultra-thin (10 nm thick) superconducting micro-bridge coupled with a planar antenna was demonstrated. However, the limitation on mixing operation of HEB is observed causing by aging of thinner layer and nano bridge structure. Tonouchi et al [Tonouchi et al, 2005] have reported extensively on the use of YBCO based antennae for $\mathrm{THz}$ detection. There are many other reports on the use of high Tc superconductors as bolometers, but only a few are listed [Du et al, 2011; Aurino et al., 2010; Laviano et al., 2010; Jagtap et al., 2009]

There are some disadvantages of bolometric type of detectors, such as they are extremely sensitive to background radiation, temperature fluctuation, mechanical vibration and electrical interference, and the performance deteriorates with increasing frequencies in the $\mathrm{THz}$ range. Further, bolometers are insensitive to phase which does not allow the reconstruction of the pulse shape in the time domain. It should be noted that background radiation defines the limiting condition of sensitivity of detector. The sensitivityof the bolometer is mainly determined by the system noise temperature. System noise includes noise from the input source and noise generated in the receiver.

\subsection{Schottky barrier detectors}

Schottky-diodes have been the only available technology for the detection of $\mathrm{THz}$ radiation for a long time \{Roeser et al, 1994]. They do not require cooling to cryogenic temperatures and cover a wide frequency range up to several THz. The main disadvantages are the poor sensitivity and the high local oscillator (LO) power requirement. The LO is still a critical element for heterodyne detection in the $\mathrm{THz}$ frequency range. Solid state sources often do not provide enough power for the heterodyne operation in the $\mathrm{THz}$ range but are under development right now. One of the early reports on Schottky diodes for THz applications was by Suzuki etal [Suzuki et al 1999]. They presented results on submicrometer Pt/GaAs diode fabrication process that resulted in a significant reduction in low-frequency noise. Noise performance was optimized by establishing an even distribution of gallium and arsenic (stoichiometric surface) at the contact surface. Diodes fabricated with the optimized procedure exhibited a signal-to-noise ratio that was better than that of commercially available diodes at $0.9 \mathrm{THz}$. Rinzan et al [Riznan et al., 2005] demonstrated a heterojunction interfacial work function internal photoemission (HEIWIP) detector with a threshold frequency $\left(f_{0}\right)$ of $2.3 \mathrm{THz}\left(\lambda_{0}=128 \mu \mathrm{m}\right)$. The threshold limit of $\sim 3.3 \mathrm{THz}(92 \mu \mathrm{m})$ was surpassed using AlGaAs emitters and GaAs barriers. The peak values of responsivity, quantum efficiency, and the specific detectivity at $9.6 \mathrm{THz}$ and $4.8 \mathrm{~K}$ for a bias field of $2.0 \mathrm{kV} / \mathrm{cm}$ are $7.3 \mathrm{~A} / \mathrm{W}, 29 \%, 5.3 \times 10^{11}$, respectively. Yasui et al [Yasui et al, 2006] developed a wide-bandwidth, high-sensitivity, continual terahertz-wave sensor that utilizes a quasioptical parabolic mirror and a Schottky barrier diode and successfully applied it at up to $7 \mathrm{THz}$ range. This sensor utilized a parabolic cylindrical mirror, a long-wire antenna, and a Schottky barrier diode. The antenna, placed at the focal point of the parabolic mirror, quasioptically collects the terahertz signalleading to superior performance. Ryzhii et al., [Ryzhii et al., 2006] investigated the plasma oscillations in a two-dimensional electron channel with a reverse-biased Schottky junction. They show that the plasma instability can 
be used in a novel diode device - lateral Schottky junction tunneling transit-time terahertz oscillator. Magno et al grew [Magno et al., 2008] Antimonide-based $p^{+} \mathrm{N}$ junctions. The diodes had good rectification with ideality factors near 1 , and high saturation current densities of $2.5 \times 10^{-2} \mathrm{~A} / \mathrm{cm}^{2}$. A cutoff frequency of $6.5 \mathrm{THz}$ is estimated from the $R C$ product. The high saturation current indicates that this diode could be used as a terahertz mixer. Maestrini et al [Maestrini et al, 2010] reviewed some aspects of the technology of terahertz heterodyne receiver front-ends dedicated to astrophysics, planetary and atmospheric sciences with focus on frequency multipliers and Schottky mixers. It is clear from this review that there is much potential for research in the area of novel architectures of power-combined frequency multipliers at submillimeter-wavelengths, $\mathrm{THz}$ planar fundamental mixers, and integrated receivers and the fabrication of submillimeter-wave planar Schottky diodes. Chen et al. [Chen et al, 2010] demonstrated high performance nanometer $\mathrm{NiSi}_{2}-\mathrm{Si}$ Schottky barrier diode arrays (SBDA) with various isolation designs, including poly Si gate (PSG) and resist protection oxide (RPO) for advanced radio frequency applications. The SBDAs could achieve a cutoff frequency of up to $4.6 \mathrm{THz}$.

It is evident from a brief survey of literature that Schottky diode structures have great potential for application in $\mathrm{THz}$ detectors impacting a variety of fields. However, there are several challenges in design, fabrication and operation of these device structures that remain unresolved

\subsection{Field-effect transistor detectors}

A channel of a Field Effect Transistor (FET) can act as a resonator for plasma waves. The plasma frequency of this resonator depends on its dimensions and for gate lengths of a micron and sub-micron (nanometer) size, can reach the Terahertz (THz) range \{Dyakanov, 2008]. The interest in the applications of FETs for THz spectroscopy started at the beginning of 90s with the work of Dyakonov and Shur [Dyakanov and Shur, 1996] who predicted that plasma oscillations in a FET channel can lead to the THz emission. Recently, non-resonant plasma properties were successfully used for the room temperature broadband $\mathrm{THz}$ detection and imaging by silicon FETs. Both THz emission and detection, resonant and nonresonant, were observed experimentally at cryogenic, as well as at room temperatures, clearly demonstrating effects related to the excitation of plasma waves. The possibility of the detection is due to nonlinear properties of the transistor, which lead to the rectification of an ac current induced by the incoming radiation. As a result, a photoresponse appears in the form of dc voltage between source and drain which is proportional to the radiation power (photovoltaic effect). There are three distinct regions of operation of the FETs depending on gate lengths. It has been demonstrated that for gate lengths of the order of $0.1 \mu \mathrm{m}$ the plasma oscillations will be in the low THZ region [Dyakanov, 2008]. Otherwise the FET can operate as a broad-band $\mathrm{THz}$ detector. There are a few other reports on the use of FETs as $\mathrm{THz}$ detectors, signifying again the potential of these structures as wellas the number of fabrication issues that need to be overcome [Knap et al., 2011]. The use of nitride based FETs for THz detection has also been demonstrated.[Shur, 2007]

\subsection{High electron mobility transistor detectors}

Semiconductors heterostructure devices such as high electron mobility transistor (HEMT) and heterojunction bipolar transistors have attracted increasing attention in $\mathrm{THz}$ devices 
[Dyakonov 2005; Fatimy 2005; Knap 2004; Lusakowski 2005; Otsuji et al., 2010; Tonouchi 2007; Tredicucci 2005], which in fact, can be regarded as a new type of terahertz detectors. Otsuji et al. [Otsuji et al., 2010] reviewed recent advances in emission of terahertz radiation from 2D electron systems in semiconductor heterostructures. A self-consistent spatiotemporal variation of quantum confined electron gas with sufficiently high electron mobility in the channel of HEMT has been proved to be an efficient mechanism for the detection and emission of terahertz radiations. The main advantage of the HEMT over the non-laser based sources is in working temperature and hence hardware requirement for operation. Another major advantage of HEMT terahertz emitters are their high tunability and low fabrication cost. The introduction of closely-spaced heterojunction interfaces under the right conditions produces a two-dimensional electron gas (2 DEG). This low dimensional plasmon (electron gas) is favourable for solid-state far-infrared and $\mathrm{THz}$ sources. The strong confinement of charge carriers in the quantum well is believed to be the cause of large electron mobility. Further, the small channel length of the device, leading to an increase in transconductance with an increase in carrier mobility, is well known for HEMT. However, the high losses caused by free carrier absorption and small confinement factor of heterostructure are the factors which restricts use of HEMT at longer $\mathrm{THz}$ wavelengths. Monte Carlo simulation performed to study of transient electron transport in wurtzite GaN, InN, and AlN shown that, for ideal transistors with $\mathrm{L}=0.1 \mu \mathrm{m}$, by replacing the $\mathrm{GaN}$ channel with InN, can be expected increase frequency of operation from 0.48 to $0.68 \mathrm{THz}$. Moreover, low $m_{e}$ (effective electron mass) in the InN channels also gives a prospect for electron nonstationary dynamics, and operating frequency over $1 \mathrm{THz}$ has been predicted for $0.1 \mu \mathrm{m}$ gates in that case [Foutz et al., 1999]. Further increase in operating frequency (THz range) is, in fact, expected in ultrathin gate structured HEMT. Fatimy et al. [Fatimy et al., 2010] have demonstrated room temperature terahertz generation by a submicron size AlGaN/GaN-based high electron mobility transistor. They have showed that these transistors are tunable by the gate voltage between 0.75 and $2.1 \mathrm{THz}$. It is also possible to tune operating frequency by manipulating cap, window, and gate lengths and other structural parameters [Ryzhii et al., 2006].

Understanding the physics of plasma wave resonant line broadening is essential to improve performance of HEMT based plasma wave detectors of terahertz radiation. Properties of HEMT terahertz detectors are defined by plasma wave excitations of two dimensional electron gas [Tsaur \& Wang, 2009]. Recently it has been demonstrated that narrow terahertz

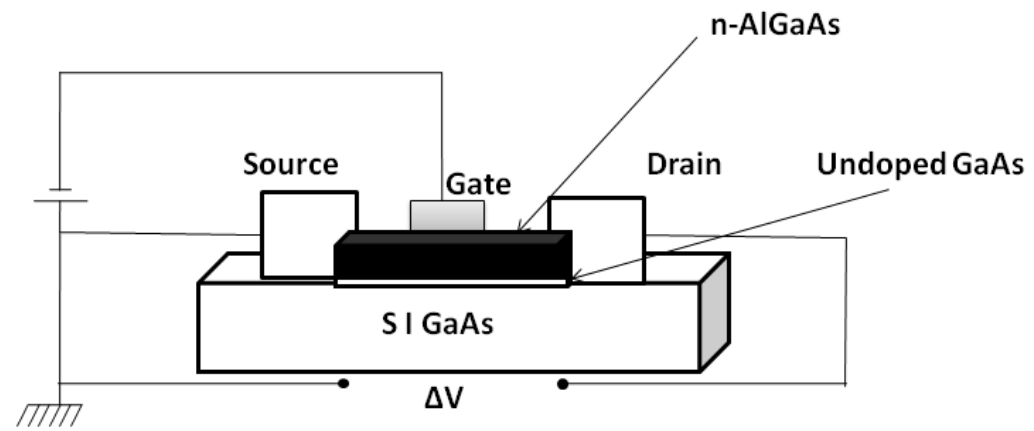

Fig. 3. The typical schematic view of a high electron mobility transistor structure 
plasma wave resonant detection at low temperature in $200 \mathrm{~nm}$ gate length InGaAs/InAlAs multichannel HEMT. To achieve selective resonant and voltage tunable terahertz emission, they have maintained the gate width is in the order of the gate length [Shchepetov et al., 2008].The typical schematic of a High electron mobility transistor used for $\mathrm{THz}$ detection is shown in fig.3.

\subsection{Sensors}

The state of technology in $\mathrm{THz}$ sensors is briefly reviewed. A variety of $\mathrm{THz}$ sensors have been developed recently mainly for use in biological and medical applications . Federici et al have reviewed the developments in $\mathrm{THz}$ sensing [Federici et al, 2005] technology, spectroscopy and imaging for security applications. The typical view of a $\mathrm{THz}$ imaging set up is shown in fig.4. The authors state that since, (a) THz radiation can detect concealed weapons since many non-metallic, non-polar materials are transparent to $\mathrm{THz}$ radiation; (b) target compounds such as explosives and illicit drugs have characteristic $\mathrm{THz}$ spectra that can be used to identify these compounds and (c) THz radiation poses no health risk for scanning of people stand-off interferometric imaging and sensing for the detection of explosives, weapons and drugs is critical. Future prospects of $\mathrm{THz}$ technology are discussed. Nagel et al [Nagel et al, 2003] first reported a novel resonant THz sensor for the label-free analysis of DNA molecules. The sensor allows the direct detection of DNA-probe molecules at functionalized electrodes via hybridization. Subsequent time resolved photoconductive sampling of the $\mathrm{THz}$ transmission identifies the binding state between probe and target DNA. Integrating neighbouring sensors on a chip, this technique can be extended to a parallel analysis of multiple DNA sequences. Nagel et al [Nagel et al, 2006] then reviewed the state of biological $\mathrm{THz}$ technology applications and conclude that success in this area will strongly depend on the development of compact, low-cost and flexible systems. They have reported on different approaches for $\mathrm{THz}$ biosensor systems based on femtosecond lasers and discussed the technology for generation, transmission and detection of $\mathrm{THz}$ signals as well as their application formarker-free biomolecule detection on functionalized

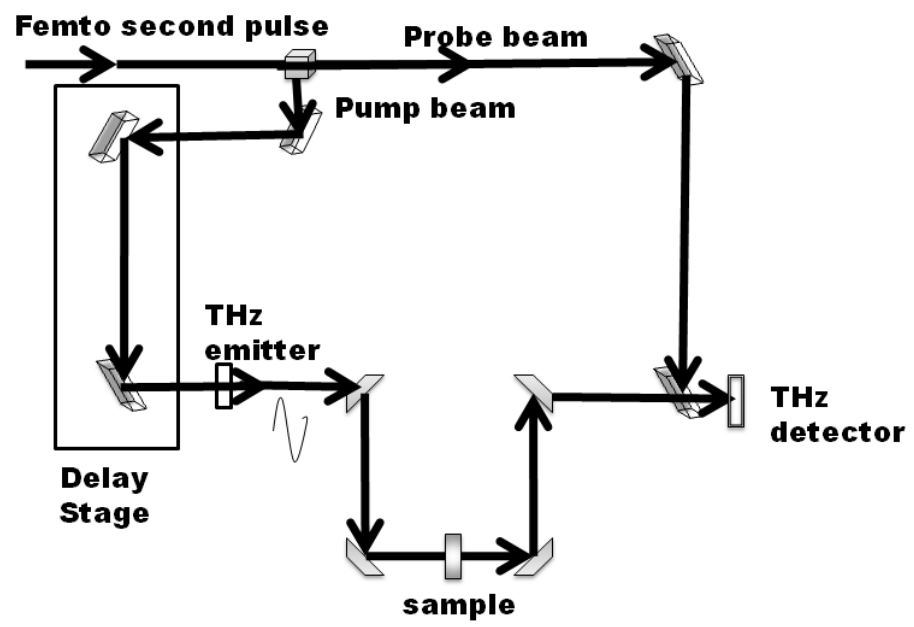

Fig. 4. Typical schematic view of a THz imaging set up. 
surfaces in dry and fluid environments. Kitagawa et al [Kitagawa et al , 2006] demonstrated the $\mathrm{THz}$ spectroscopic performance of highly integrated sensor chips based on microstrip lines by measuring biomolecule/water systems. The concentration resolution of the chips reaches down to $0.05 \mathrm{~g} / \mathrm{ml}$. They have confirmed that the number of bound water molecules per biomolecule can be obtained with precision using solid state transmission lines. The chips are highly suitable for the inspection of small amounts of specimen and for the application to a wide range of water rich materials. McLaughlin et al [McLaughlin et al , 2008] employed poled polymer films $10-15 \mu \mathrm{m}$ thick with electro-optic coefficients as high as $160 \mathrm{pm} / \mathrm{V}$ at $1300 \mathrm{~nm}$ are used to generate and sense subpicosecond pulses with continuous bandwidth up to $15 \mathrm{THz}$. The use of the poled polymer as the terahertz sensor for the identification of DAST phonons at 1.1, 3.0, 5.3, 8.5, and $12.5 \mathrm{THz}$ was demonstrated. Kawase etal [Kawase etal , 2010] have suggested a wide range of real-life applications using novel terahertz imaging techniques. A high-resolution terahertz tomography has been demonstrated by ultra short terahertz pulses using optical fiber and a nonlinear organic crystal. They, further, describe a nondestructive inspection system that can monitor the soot distribution in a ceramic filter using millimeter-to-terahertz wave computed tomography. Another proposed application is in the area of thickness measurement of very thin films using the high-sensitivity metal mesh filter. Abbas et al [Abbas et al , 2009] describe the development, functionalization and functionality testing of a TeraHertz (THz) BioMicroElectroMechanical System (BioMEMS) dedicated to enzyme reaction analysis. The microdevice was fabricated by mixing clean room microfabrication with cold plasma deposition. The progression of the hydrolysis reaction over time was monitored by the $\mathrm{THz}$ sensor connected to a vectorial network analyzer. Preliminary results showed that sub- $\mathrm{THz}$ transmission measurements are able to discriminate different solid films, various aqueous media and exhibit specific transmission behavior for the enzyme hydrolysis reaction in the spectral range 0.06-0.11 THz. Rau et al [Rau et al., 2005] reported on a simple $\mathrm{THz}$ waveguide element used as a sensitive sensor for adsorbates on surfaces. The evanescent wave from total internal reflection off a silicon prism was used to couple pulsed $\mathrm{THz}$ radiation frequency selectively into the waveguide. The coupled frequencies were determined via time domain spectroscopy and react sensitively to any changes of thickness or phase shift upon reflection. In particular, the sensitivity to phase shifts makes this waveguide sensor attractive for the detection of very thin adsorbates. Xiao-li and Jiu-sheng [Xiao-li \& Jiu-sheng,2011] used terahertz time-domain spectroscopy technique to test flour/talc powder mixture samples. They found that the two samples have distinct absorption peaks and different refractive index in the $\mathrm{THz}$ region leading to the possibility of $\mathrm{THz}$ applications to the field of food safety. Mendis et al [Mendis et al, 2009] describe a terahertz optical resonator suited for highly sensitive and noninvasive refractive-index monitoring. The resonator is formed by machining a rectangular groove into one plate of a parallel-plate waveguide, and is excited using the lowest-order transverse-electric $\left(\mathrm{TE}_{1}\right)$ waveguide mode. Since the resonator can act as a channel for fluid flow, it can be easily integrated into a microfluidics platform for real-time monitoring. Using this resonator with only a few microliters of liquid, a refractive-index sensitivity of $3.7 \times 10^{5} \mathrm{~nm} /$ refractive-indexunit was demonstrated in the $\mathrm{THz}$ range. $\mathrm{Lu}$ et al [Lu et al., 2008] discuss broadband terahertz wave detection through field-induced second harmonic generation using selected gases. The dependences of the detected second harmonic intensity on probe pulse energy, bias field strength, gas pressure, and third order nonlinear susceptibility are systematically investigated with xenon, nitrogen, $\mathrm{SF}_{6}$, and alkanes. Experiment results reveal that the 
detected second harmonic intensity quadratically depends on the third order nonlinear susceptibility of the gas. Two orders of magnitude enhancement in the dynamic range of broadband terahertz wave detection are observed with alkane gas $\left(\mathrm{C}_{4} \mathrm{H}_{10}\right)$ sensor. Foltynowicz et al [ Foltynowicz et al, 2006] reported the vapor-phase spectrum of 2,4dinitrotoluene (DNT) from $0.05 \mathrm{THz}$ to $2.7 \mathrm{THz}$ utilizing pulsed terahertz time-domain spectroscopy. They observed a broad-band absorption profile from $50 \mathrm{GHz}$ to $600 \mathrm{GHz}$, peaking at $240 \mathrm{GHz}$. This broad absorption profile corresponds to DNTs pure rotational spectrum, which was confirmed by asymmetric top model calculations. Superimposed on the broad absorption profile, we observed discrete structure that extended to the higher $\mathrm{THz}$ frequencies. Leahy-Hoppa et al [Leahy-Hoppa et al, 2007] have shown Time-domain terahertz spectroscopy (TDTS) to be a promising tool in detection of explosives and explosive related compounds. The $\mathrm{THz}$ absorption spectra over an extended frequency band from 0.5 to $6 \mathrm{THz}$ were reported for four explosives: RDX (1,3,5-trinitroperhydro-1,3,5triazine), HMX (1,3,5,7-tetranitroperhydro-1,3,5,7-tetrazocine), PETN (pentaerythritol tetranitrate), and TNT (2,4,6-trinitrotoluene). New distinctive spectral features are shown in these materials between 3 and $6 \mathrm{THz}$. Zhang et al [Zhang et al, 2008] present terahertz reference-free phase imaging for identification of three explosive materials (HMX, RDX, and DNT). They propose a feature extraction technique to locate the spectral position of an unknown material's absorption lines without using the reference signal. The samples are identified by their absorption peaks extracted from the negative first-order derivative of the sample signal phase divided by the frequency at each pixel. Hu etal [ $\mathrm{Hu}$ et al, 2006] report experimental measurement and theoretical analysis of $\mathrm{THz}$ spectrum for five different explosives and related compounds are introduced. The refractive index and absorption coefficient of the samples are measured in the region of 0.2-2.6 terahertz by time-domain spectroscopy. The simulated spectrum of $\gamma$-HNIW is in agreement with the experimental data.

It is thus evident that there are many significant developments in the area of $\mathrm{THz}$ sensors that impact various aspects of biology, medicine and security.

\subsection{New materials and device structures}

A large variety of new types of materials and device structures have been proposed recently for $\mathrm{THz}$ applications. These include the use of carbon nanotubes [Costa et al., 2009], GaAs based nanotransistors [Łusakowski, 2007] and polar optical phonons in quantum wells [Liu et al., 2011].Sputter deposited zinc oxide photoconductive antenna have also been proposed for $\mathrm{THz}$ detection applications[Iwami et al., 2009]. Videlier et al have demonstrated Si MOSFET structures as $\mathrm{THz}$ detectors[Videlier et al].The possibility of utilizing twodimensional plasmons in semiconductor heterostructures for $\mathrm{THz}$ detection has been also been proposed [Otsuji et al, 2010]. Cox et al [Cox et al., 2011\} demonstrated a MEMS based uncooled $\mathrm{THz}$ detector. The development of novel single photon detectors that work in the range of 10-50um based on double quantum weel structures have been reported recently \{Ueda\& Komiyama, 2010]. There are many other reports on novel materials and structures, these are however beyond the scope of the current review.

\section{ZnTe coatings for $\mathrm{THz}$ applications}

In this section we present a review of the work being carried out by the present authors in the area of $\mathrm{THz}$ materials development. The ability to miniaturize $\mathrm{THz}$ photonics depends 
critically on two developments compact $\mathrm{THz}$ sources and elimination of the dependence on crystals as emitters and detectors. The focus is, therefore, mainly on the development of thick ZnTe coatings for use in $\mathrm{THz}$ emitter and detector applications, based on the principle of optical rectification. As stated in section 3.2, optical rectification can be controlled by manipulating the crystal structure and orientation. It is, however, a weak non-linear response. Hence, material thicknesses have to be high to generate a measurable response. Semiconductors from the II-VI group, especially ZnTe crystals [Turchinovich \& Dijkhuis, 2007; ], have drawn considerable interest in recent years for THz applications. ZnTe is intrinsically a p-type semiconductor from the II-VI family with a direct bandgap of $2.26 \mathrm{eV}$. The growth of $n$ type ZnTe is still a challenge, which must be overcome before $p-n$ junction devices can be realized. Incorporation of excess $Z n$ during growth may increase donor concentration favoring the formation of $n$-type ZnTe. The more commonly encountered polymorph of ZnTe is the zinc blende structure which has four asymmetric units in its unit cell as against two in the wurtzite form. The higher degree of asymmetric coordination in zinc blende ZnTe favors formation of non-centrosymmetric structure which is very useful in terahertz generation and detection applications [4]. The applications of wurtzite ZnTe, on the other hand, are less well reported. Information on properties like spectral transmission, refractive index, optical band gap, and electronic structure of wurtzite ZnTe thin films is very sparse.

Since most of the THz related work on ZnTe is on single crystals, the main objectives of our work is to fabricate ZnTe coatings that are 10-12 microns thick as well as stabilizing the wurtzite form of ZnTe and investigate its optical and electronic properties. The coatings were produced by either thermal or electron beam evaporation in high vacuum (10-6 Torr). The source material is zinc blende or wurtzite ZnTe. The substrates are borosilicate glass or fused silica and the films were deposited at room temperature. Spectral transmittance curves of the films in the wavelength range from 190 to $2500 \mathrm{~nm}$ were measured using a spectrophotometer and from these spectra the refractive index and optical band gaps were extracted. Crystal structure was determined suing a powder x-ray diffractometer and electron diffraction while microstructure and morphology was examined under an Atomic force microscope.

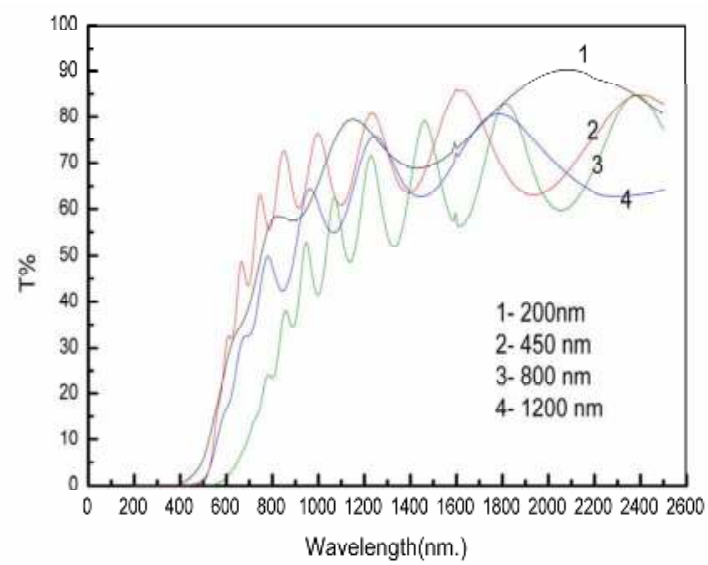

Fig. 5. Spectral transmission curves 


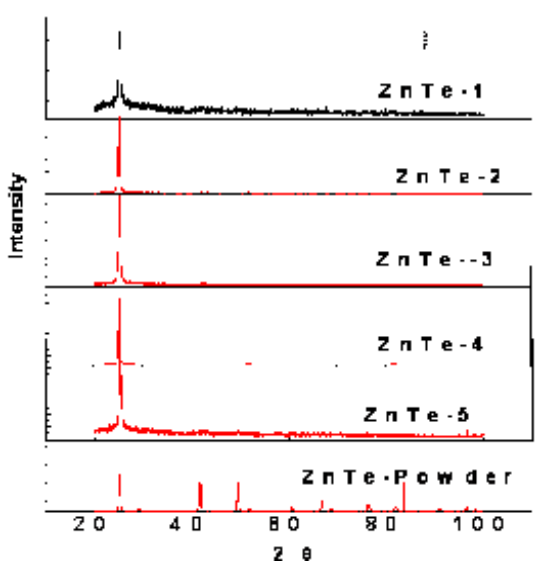

Fig. 6. X-ray diffraction patterns of the ZnTe films of different thicknesses showing the formation of zinc blende structure.

The measured spectral transmission curves for zinc blende ZnTe films of thicknesses upto 1200 $\mathrm{nm}$ and typical x-ray diffraction pattern of the $1200 \mathrm{~nm}$ film is shown in figs. 5 and 6 respectively. The transmission of the films is close to $90 \%$ in the near IR region and calculated refractive indices were between 2.0 and 2.6. The optical band gaps were between 2 and $2.2 \mathrm{eV}$. These values compared favourably with earlier reports.Significantly the $1200 \mathrm{~nm}$ thick film was nanocrystalline ( with a crystallite size of $24 \mathrm{~nm}$ ) and showed a preferred (111) orientation. The thickest films achieved using this technique had a thickness of 10-12 microns

The atomic force microscope image of a $2000 \mathrm{~nm}$ thick zinc blende ZnTe film is shown in fig. 7. The film is clearly very smooth with roughness less than $10 \mathrm{~nm}$ even at this thickness. The grain sizes are of the order of $100 \mathrm{~nm}$.

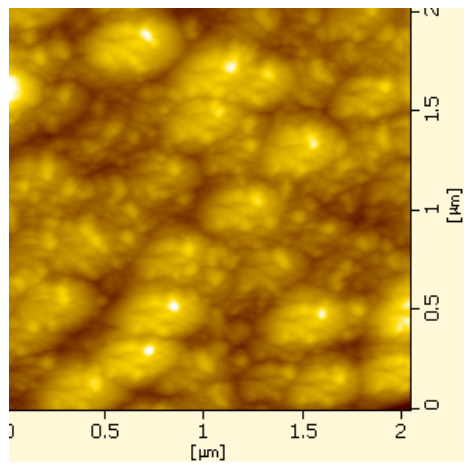

Fig. 7. Atomic force microscope image of a $2000 \mathrm{~nm}$ zinc blende ZnTe film.

ZnTe also crystallizes in the wurtzite structure wherein the stacking sequence of the cation and anion is $A B A B A B A B$. . . with each repeated period perpendicular to the basal plane, whereas in the zinc blende structure the sequence is $A B C A B C A B C$. Therefore, the distance between the third nearest-neighbor (NN) atoms is small in wurtzite ZnTe. In contrast, the distance between the first and second NN atoms is invariable in both structures. In addition, 
the Zn-Te bond in zinc blende ZnTe has greater ionic character than that in wurtzite ZnTe. Due to these differences it has been shown from ab-initio calculations that the band gaps of the structures should be different. Similarly, it is also expected that the electronic properties will be different. There are, however, very limited studies on the growth and stabilization of wurtzite ZnTe films. The present authors have grown wurtzite ZnTe films by electron beam evaporation of a wurtzite ZnTe source material. Typical x-ray diffraction patterns of the wurtzite films are shown in fig.8 for different thicknesses. The measured spectral transmittance for the zinc blende and wurtzite films are shown in fig.9. Detailed dispersion analysis of the films and comparison with zinc blende films has been carried out. ZnTe thin films exhibit either the wurtzite or zinc blende structure depending on the chemical nature of the source material. The formation of the structural phases is confirmed by a combination of x-ray diffraction and selected area electron diffraction. The films were nanocrystalline and randomly textured. The refractive index of the wurtzite phase (3.87 at $2000 \mathrm{~nm}$ ) is higher than that for the zinc blende phase (3.03 at $2000 \mathrm{~nm})$. The optical band gap, however, is lower for the wurtzite phase $(1.1 \mathrm{eV})$ than the zinc blende phase $(2.26 \mathrm{eV})$. The optical dispersion behavior has been investigated within the framework of the single effective oscillator model. It is shown that the oscillator strength, dispersion energy and optical charge carrier concentrations are all lower for the wurtzite phase than the zinc blende phase.

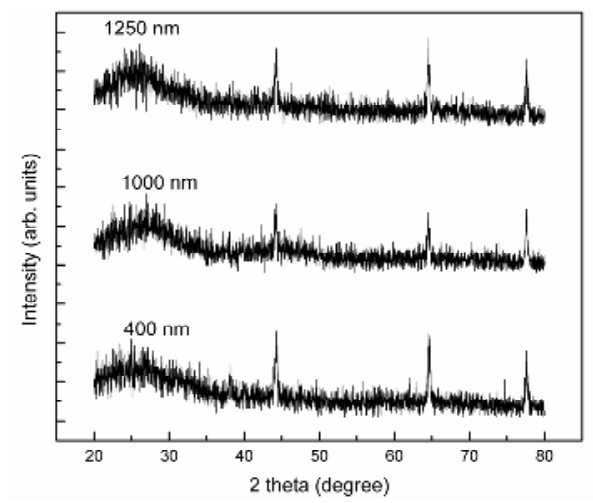

Fig. 8. XRD patterns of wurtzite ZnTe films of different thickness

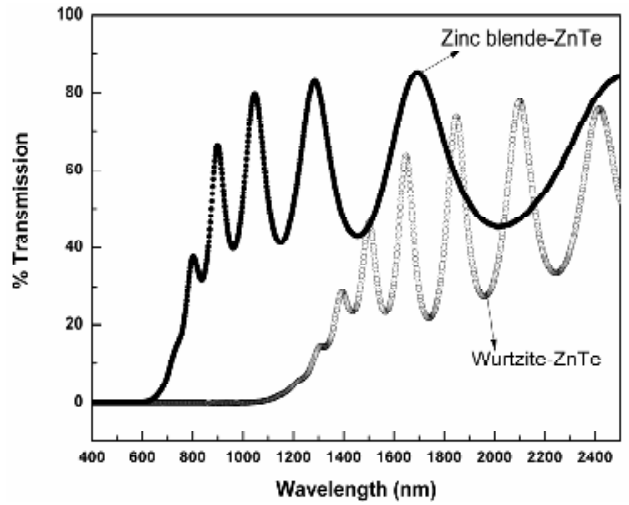

Fig. 9. Comparison of optical response of zinc blende and wurtzite ZnTe thin films. 
The charge carrier concentration in wurtzite phase $\left(5 \times 10^{25} \mathrm{~m}^{-3}\right)$ is lower than the zinc blende structure $\left(4.17 \times 10^{26} \mathrm{~m}^{-3}\right)$ This may make the wurtzite phase suitable for the development of $n$-type ZnTe, which has been a long standing challenge. Evidently the wurtzite phase has the potential to be employed as an n-type material leading to the formation of ZnTe p-n junctions for $\mathrm{THz}$ applications. Further developments in this direction are ongoing

Our work thus demonstrates the growth of thick ZnTe coatings in both the zinc blende and wurtzite polymorphs. Their optical properties are substantially different, signifying the possibility of applications in different frequencies regimes of the $\mathrm{THz}$ region [Kiran et al, 2010; Kshirsagar et al, 2011].

\section{Conclusions and future prospects}

In summary, a number of technologies for $\mathrm{THz}$ emitters, detectors and sensors have been reviewed. It is obvious that, while much progress has been made in all three areas, there is scope for new science and technology to emerge in all these areas. Significantly, the progress in the area of $\mathrm{THz}$ electronics has been much more rapid than that in the area of $\mathrm{THz}$ photonics. This is mainly due to the lack of cost effective so-called "table top" femto second laser sources. $\mathrm{THz}$ electronics, on the other hand, relies solely on existing mature technologies for materials and device fabrication. Much of the materials development has focused on single crystals. However, for miniaturization of $\mathrm{THz}$ technologies (Photonics and electronics) to succeed, the area of coatings, films and micro-nano devices has to be focused upon, in addition to development of compact THz sources. A report on the development of a high-resolution terahertz $(\mathrm{THz})$ imaging beyond the diffraction limit by using a twodimensional electron gas (2DEG) in a GaAs/ AlGaAs heterostructure, points to effort in this direction[.Kawano \& Ishibashi, 2010]. In the area of materials, strongly correlated electron systems have shown great potential[Ge et al, 2010]. In addition, novel device structures to exploit the plasma instability for $\mathrm{THz}$ emission and detection is another area that is receiving greater attention. Interestingly, access to fs laser sources has led to better understanding of physical and chemical phenomena in the THz region, that will eventually lead to new materials and device structures. Two examples exemplify this theme. It has recently been shown [Chan et al., 2011] that a nano mechanical oscillator coupled to single mode electromagnetic oscillator in a nano optical cavity can be designed to have oscillations in $\mathrm{THz}$ frequency domain. The physics of this domain of energy storage as envisaged by the Debye model has to be examined and the modes of vibrations at phase boundaries, and domain boundaries studied through $\mathrm{THz}$ experiments. These will reveal new materials and methods of generation and detection and sensing in the $\mathrm{THz}$ range. In another report [Chen et al, 2011], impulsive stimulated Raman scattering and Time-domain $\mathrm{THz}$ spectroscopy of coherent optical phonons in bismuth germinate $\left(\mathrm{Bi}_{4} \mathrm{Ge}_{3} \mathrm{O}_{12}\right)$ revealed more than 12 unique vibrational states ranging in frequency from 2 to $11 \mathrm{THz}$, each with coherent lifetimes ranging from 1 to 20 ps. Such studies on other systems can be expected have a profound impact on the developments in THz sources, emitters, detectors and sensors.

\section{Acknowledgements}

The authors acknowledge the facilities provided by the School of Physics under the UGCUPE and CAS programs. A Dr D S Kothari Fellowship for SK is also acknowledged. 


\section{References}

Abbas, A.; Treizebre, A.; Supiot, P.; Bourzgui, N.; Guillochon, D.; Vercaigne-Marko, D. \& Bocquet, B. (2009). Cold plasma functionalized TeraHertz BioMEMS for enzyme reaction analysis, Biosensors and Bioelectronics, Vol.25, No.1, (September 2009), pp. 154-160, ISSN 09565663

Aurino, M., Kreisler, A.J., Türer, I., Martinez, A., Gensbittel, A., Dégardin, A.F. (2010) Journal of Physics: Conference Series Vol.234, No.4 (2010) art. no. 042002. ISSN: 1742-6588.

Belkin, M. A.; Jie, W. Q.; Pflugl, C.; Belyanin, A.; Khanna, S. P. Davies, A. G.; Linfield, E. H. Capasso, F. (2009). High-Temperature Operation of Terahertz Quantum Cascade Laser Sources, IEEE Selected Topics in Quantum Electronics, Vol.15, No.3 (June 2009) pp. 952-967, ISSN 1077-260X

Bhasin L. \& Tripathi, V. K. (2009). Terahertz generation via optical rectification of $x$-mode laser in a rippled density magnetized plasma, Phys. Plasmas Vol.16, No. 10, (October 2009), pp. 103105- 1-6 ISSN 1089-7674

Blanchard,F.; Sharma, G.; Razzari, L.; Ropagnol, X.; Bandulet, H -C.; Vidal, F.; Morandotti, R.; Kieffer, J -C.; Ozaki, T.; Tiedje, H.; Haugen, H.; Reid, M. \& Hegmann, F. (2011). Generation of Intense Terahertz Radiation via Optical Methods, IEEE Selected Topics in Quantum Electronics, Vol.17 No. 1, (June 2011), pp. 5-16, ISSN 1077-260X

Bolivar P H, Nagel M, Richter F, Brucherseifer M, Kurz H, Bosserhoff A \& Buttner R (2004), Label-free THz sensing of genetic sequences: towards 'THz biochips', Philosophical Transactions of the Royal Society, London, A., Vol. 362 (2004) pp.323-335.

Bugay, A. N. \& Sazonov, S. V. (2010). The generation of terahertz radiation via optical rectification in the self-induced transparency regime, Phys. Lett. A, Vol.374, No. 8 (February 2010), pp. 1093-1096, ISSN 0375-9601

Caumes, J. P.; Chassagne, B.; Coquillat, D.; Teppe, F. \& Knap, W. (2009). Focal-plane microbolometer arrays for $0.5 \mathrm{THz}$ spatial room-temperature imaging, IEEE Electronics Letters, Vol.45, No. 1, (December 2009), pp. 34-35, ISSN 0013-5194

Chan J., Mayer Alegre T.P., Safavi-Naeini A.H.,, Hill J.T., Krause A, Groblacher S., Aspelmeyer M \& Painter O, (2011), Laser cooling of a nanomechanical oscillator into its quantum ground state, Nature, Vol. 478, (October, 2011), pp. 89-92. ISSN: 0028-0836.

Chamberlain J.M., (2004) Where optics meets electronics: recent progress in decreasing the terahertz gap, Philosophical Transactions of the Royal Society, London, A., Vol. 362 (2004) pp.199-213. ISSN: 1471-2962.

Chen, J.; Liang, M.; Kang, L.; Jin, B. B.; Xu, W. W.; Wu, P. H.; Zhang, W.; Jiang, L.; Li, N. S. \& Shi, C. (2009). Low Noise Receivers at $1.6 \mathrm{THz}$ and $2.5 \mathrm{THz}$ Based on Niobium Nitride Hot Electron Bolometer Mixers, Appl. Superconductivity, IEEE Trans. Vol.19, No. 3, (July 2009), pp. 278-271, ISSN 1051-8223

Chen, S.-M.; Fang, Y.-K.; Juang, F.R.; Yeh, W.-K.; Chao, C.-P. \& Tseng, H.-C (2010). Terahertz Schottky barrier diodes with various isolation designs for advanced radio frequency applications, Thin Solid Films, Vol.519, No.1, (October 2010), pp. 471-474, ISSN 0040-6090.

Chen, Z.; Gao, Y.; \& DeCamp, M. F. (2011). Retrieval of terahertz spectra through ultrafast electro-optic modulation, Appl. Phys. Lett., Vol.99, No.1, (July 2011), pp. 011106-09 ISSN 1077-3118

Cherednichenko S., Khosropanah P., Kollberg E., Krong M., Merkel H., (2002), Terahertz superconducting hot electron bolometer mixers, Physica C; Superconductivity, Vol. 372-376, pt. 1, (August 2002), pp. 407-415. 
Coquillat, D., Teppe, F., Videlier, H., Coquillat, D., Lusakowski, J., Skotnicki, T. Silicon field effect transistors for Terahertz detection and imaging Knap, W., Shuster, F., (2011) Proceedings of the 5th European Conference on Antennas and Propagation, EUCAP 2011, art. no. 5782258, (2011) pp. 3180-3182.

Costa M. R., Kibis O.V., \& Portnoi M.E.(2009), Carbon nanotubes as a basis for terahertz emitters and detectors, Microelectronics Journal , Vol. 40 (2009) 776-778

Cox, J.A., Higashi, R., Nusseibeh, F., Zins, C. (2011) MEMS-based uncooled THz detectors for staring imagers Proceedings of SPIE - The International Society for Optical Engineering 8031, (2011) art. no. 80310D.

Du, J., Hellicar, A.D., Hanham, S.M., Li, L., MacFarlane, J.C., Leslie, K.E., Foley, C.P. (2011) YBCO hot-electron bolometers dedicated to $\mathrm{THz}$ detection and imaging: Embedding issues Journal of Infrared, Millimeter, and Terahertz Waves Vol. 32 No.5, (2011) pp. 681-690.

Dyakonov M. I,.(2010) Generation and detection of Terahertz radiation by field effect transistors Comptes Rendus Physique, Vol. 11 (2010) pp. 413-420

Dyakonov, M.; \& Shur, M. (1996). Detection, mixing, and frequency multiplication of terahertz radiation by two-dimensional electronic fluid, (August 1996), Electron Devices, IEEE Trans, Vol.43, No.3, (1996) pp. 380-387, ISSN 0018-9383

Dyakonova, N.; Teppe, F.; Lusakowski, J.; Knap, W.; Levinshtein, M.; Dmitriev, A.P.; Shur, M.S.; Bollaert, S.; Cappy A. (2005). Magnetic field effect on the terahertz emission from nanometer InGaAs/AlInAs high electron mobility transistors, J. Appl. Phys. Vol.97, No.11, (May 2005), pp. 114313-1-3 ISSN 1089-7550

Fatimy, A. E.; Dyakonova, N.;, Meziani, Y.; Otsuji, T.; Knap, W.; Vandenbrouk, S.; Madjour, K.; Théron, D.; Gaquiere, C.; Poisson, M. A.; Delage, S.; Prystawko, P. \& Skierbiszewski C.; (2010). AlGaN/GaN high electron mobility transistors as a voltage-tunable room temperature terahertz sources, J. Appl. Phys. Vol.107, No.2, (January 2010), pp-024504 1-4, ISSN 1089-7550

Federici, J. F.; Schulkin, B.; Huang, F.; Gary, D.; Barat, R.; Oliveira, F. \& Zimdars, D. (2005). $\mathrm{THz}$ imaging and sensing for security applications-explosives, weapons and drugs, Semicond. Sci. Technol. Vol.20, No.7 (July 2005), pp. S266, ISSN 0268-1242

Ferguson B., Zhang X.C.,(2002) Materials for Terahertz Science and Technology, Nature Materials Vol. 1 (2002) 26-33. ISSN 1476-1122.

Foltynowicz, R. J.; Allman, R. E. \& Zuckerman, E. (2006). Terahertz absorption measurement for gas-phase 2,4-dinitrotoluene from $0.05 \mathrm{THz}$ to $2.7 \mathrm{THz}$, Chemical Physics Letters, Vol.431, No.1 (November 2006), pp 34-38, ISSN 0009-2614

Foutz, B. E.; O'Leary, S. K.; Shur, M. S. \& Eastman, L. F. (1999). Transient electron transport in wurtzite GaN, InN, and AlN, J. Appl. Phys., Vol.85, No.11 (February 1999), pp. 7727 1-8, ISSN 1089-7550

Freeman, J. R.; Brewer, A.; Beere, H. E. and Ritchie, D. A., (2011). Photo-luminescence study of heterogeneous terahertz quantum cascade lasers, J. Appl. Phys., Vol.110, No.1, (July 2011) pp. 013103 1-6, ISSN 1089-7550

Ge C., Jin K., Lu H., Wang C., Zhao G., Zhang L., Yang G., (2010), Mechanisms for the enhancement of the lateral photovoltage in perovskite heterostructures, Solid State Communications, Vol. 150 (2010) pp. 2114-2117

Ginzburg N. S., Malkin A.M., Yu. Peskov N., Sergeev A. S., Yu. Zaslavsky V,\& Zotova I.V.,(2011) Powerful terahertz free electron lasers with hybrid Bragg reflectors, Physical Review Special Topics: Accelerators and Beams Vol.14, No.4 (2011) 042001-1-9. 
Gmachl, C.; Belyanin, A.; Sivco, D.L.; Peabody, M.L.; Owschimikow, N.; Sergent, A.M.; Capasso, F. \& Cho, A.Y. (2003). Optimized second-harmonic generation in quantum cascade lasers, Quantum Electronics, IEEE, Vol.39 , No.11, (November 2003), pp. 1345-1355, ISSN 0018-9197

He, S.; Chen, X.; Wu, X.; Wang, G. \& Zhao, F. (2008). Enhanced Terahertz Emission From ZnSe Nano-Grain Surface, Journal of Lightwave Technology, Vol.26, No.11, (June 2008), pp. 1519 - 1523, ISSN 0733-8724

Hoffmann, M. C.; Yeh, K.; Hwang, H. Y.; Sosnowski, T. S.; Prall, B. S.; Hebling, J. \& Nelson, K. A. (2008). Fiber laser pumped high average power single-cycle terahertz pulse source, Appl. Phys. Lett. Vol.93, No. 14 (October 2008), pp. 141107 1-3 ISSN 1077-3118

$\mathrm{Hu}$, Y.; Huang, P.; Guo, L.; Wang, X. \& Zhang, C. (2006). Terahertz spectroscopic investigations of explosives, Phys. Lett. A, Vol.359, No.6, (December 2006), pp. 728732, ISSN 0375-9601

Il'in, K.S.; Semenov, A.D.; Hübers, H.-W. \& Siegel, M. (2010). Hot-electron bolometer mixers for terahertz radiation, Electron. Lett. Vol.46, No. 26, (January 2010), pp. S14-S16, ISSN 0013-5194

Innocenzi P, Malfatti L, Piccinini M, Sali D, Schade U,\& Marcelli A (2009), Application of Terahertz Spectroscopy to Time-Dependent Chemical-Physical Phenomena, Journal of Physical Chemistry A, Vol. 113, No. 34, (2009) pp. 9418-9423.

Iwami, K., Ono, T., Esashi, M. 2009,Sputter deposited zinc oxide photoconductive antenna for terahertz time-domain spectroscopy Proceedings of SPIE - The International Society for Optical Engineering Vol. 7133, (2009), art. no. 713315

Jacobsen R. H., Mittleman D. M.,\& M. C. Nuss, (1996) Chemical recognition of gases and gas mixtures with terahertz waves, Optics Letters, Vol. 21, No. 24 (December 1996) pp.2011-2013.

Jagtap V.S., Scheuring A., Longhin M., Kreisler A.J., Dégardin A.F., (2009) From superconducting to semiconducting YBCO thin film bolometers: Sensitivity and crosstalk investigations for future thz imagers IEEE Transactions on Applied Superconductivity Vol. 19, No. 3 (2009) pp. 287-292.

Kasai, S.; Katagiri, T.; Takayanagi, J.; Kawase, K. and Ouchi, T. (2009). Reduction of phonon resonant terahertz wave absorption in photoconductive switches using epitaxial layer transfer, Appl. Phys. Lett., Vol.94, No.11, (2009) pp. 113505 1-3 ISSN 1077-3118

Kawano Y., Ishibashi K. (2010) Physica E 42 (2010) 1188-1191

Kawase, K.; Shibuya, T.; Hayashi, S. \& Suizu, K. (2010). THz imaging techniques for nondestructive inspections, Comptes Rendus Physique, Vol.11, No.7, (October 2010), pp. 510-518, ISSN 1631-0705

Kiran M.S.R.N., Kshirsagar S.D., Krishna M.G., Tewari S.P. (2010), Structural, optical and nanomechanical properties of (111) oriented nanocrystalline ZnTe thin films, European Journal of Applied Physics: Applied Physics, vol. 51 (October 2010) pp.10502-9.

Kitagawa, J.; Ohkubo, T.; Onuma, M. \& Kadoya, Y., (2006). THz spectroscopic characterization of biomolecule/water systems by compact sensor chips, Appl. Phys. Lett. Vol.89, No.4, (July 2006), pp. 041114 1-3, ISSN 1077-3118

Klatt G., Hilser F., Qiao W., Beck M., Gebs R., Bartels A., Huska K., Lemmer U., Bastian G., Johnston M.B., Fischer M., Faist J., \& Dekorsy T., (2010) Terahertz emission from lateral photo-Dember currents, Optics Express, Vol. 18, Issue 5, (2010) pp. 4939-4947 
Kleinschmidt, P.; Giblin, S. P.; Antonov, V.; Hashiba, H.; Kulik, L.; Tzalenchuk, A. \& Komiyama, S. (2007). A Highly Sensitive Detector for Radiation in the Terahertz Region, IEEE trans. Instru. measurement, Vol.56, No. 2, (2007) pp. 463-467, ISSN 0018-9456

Kida N, Murakami H \& Tonouchi M., (2005) Terahertz optics in strongly correlated electron systems, Topics in Applied Physics, Vol. 97 (2005) pp. 215-334.

Knap, W.; Lusakowski, J.; Parenty, T.; Bollaert, S.; Cappy, A.; Popov, V.V. \& Shur, M.S. (2004). Terahertz emission by plasma waves in $60 \mathrm{~nm}$ gate high electron mobility transistors, Appl. Phys. Lett. Vol.84, No.13, (February 2004), pp. 2331 1-3, ISSN 1077-3118

Knap, W.; Shuster, F.; Coquillat, D.; Teppe, F.; Videlier, H.; Coquillat, D.; Lusakowski, J. \& Skotnicki, T. (2011). Silicon field effect transistors for Terahertz detection and imaging, Proceedings of the 5th European Conference on Antennas and Propagation, Antennas and propagation. european conference, April, 2011, Rome, Italy

Krotkus A. (2010), Semiconductors for terahertz photonics applications, Journal of Physics D ; Applied Physics, Vol.43 (2010) pp.273001-273006. ISSN : 0022-3727

Kshirsagar S D, Krishna M.G., Tewari S.P., (2011), Morphological and Optical properties of Wurtzite ZnTe thin films, AIP Conference proceedings, Vol. 1349 (July 2011), pp. 1285-6.

Laviano, F., Gerbaldo, R., Ghigo, G., Gozzelino, L., Minetti, B., Rovelli, A., Mezzetti, E., (2010) Rugged superconducting detector for monitoring infrared energy sources in harsh environments, Superconductor Science and Technology Vol. 23, No.12 (2010), art. no. 125008 .

Leahy-Hoppa, M.R.; Fitch, M.J.; Zheng, X.; Hayden, L.M. \& Osiander, R. (2007). Wideband terahertz spectroscopy of explosives, Chem. Phys. Lett., Vol.434, No.4 (February 2007), pp. 227-230, 0009-2614

Lewis, R.A. (2007) Physical phenomena in electronic materials in the terahertz region Proceedings of the IEEE Vol. 95 No. 8, art. no. 4337844 (2007), pp. 1641-1645.

Li C., Zhou M., Ding W., Du F., Li Y., Wang W .M., Sheng Z.M., Ma J.L., Chen L M, Dong Q, \& Zhang J, Effects of laser plasma interactions on $\mathrm{THz}$ radiation from solid targets irradiated by ultra short intense laser pulses, Physical Review E, Vol. 84 (2011) pp. 036405-036410

Li, D. \& Ma, G. (2008). Pump-wavelength dependence of terahertz radiation via optical rectification in (110)-oriented ZnTe crystal, J. Appl. Phys. Vol.103, No. 12, (June 2008), pp. 123101 1-4, ISSN 1089-7550

Liu, H.C., Song, C.Y., Wasilewski, Z.R., Buchanan, M. (2011) Phonon and polaron enhanced IR-THz photodetectors Proceedings of SPIE - The International Society for Optical Engineering 7945, (2011) art. no. 79450X

Liu, L. Xu, H.; Percy, R. R.; Herald, D. L.; Lichtenberger, A. W.; Hesler, J. L. \& Weikle, R. M. (2009). Development of Integrated Terahertz Broadband Detectors Utilizing Superconducting Hot-Electron Bolometers, IEEE trans. Appl.Supercon. Vol.19, No. 3, (July 2009), pp. 282-286, ISSN 1051-8223

Liu, T.; Lin, G.; Lee, Y.; Wang, S.; Tani, M.; Wu, H. \& Pan, C. (2005). Dark current and trailing-edge suppression in ultrafast photoconductive switches and terahertz spiral antennas fabricated on multienergy arsenic-ion-implanted GaAs, J. Appl. Phys. Vol.98, No.1, (July 2005), pp. 013711 1-4, ISSN 1089-7550

Lloyd-Hughes, J.; Castro-Camus, E.; Johnston, M.B. (2005). Simulation and optimisation of terahertz emission from InGaAs and InP photoconductive switches, Solid State Commun., Vol.136, No.11, (October 2005) pp. 595-600, ISSN 0038-1098 
Lu, X.; Karpowicz, N.; Chen, Y. \& Zhang, X.-C., (2008). Systematic study of broadband terahertz gas sensor, Appl. Phys. Lett. Vol.93, (December 2008), pp.261106, ISSN 1077-3118

Łusakowski J., (2007), Nanometer transistors for emission and detection of THz radiation, Thin Solid Films, Vol. 515 (2007) pp. 4327-4332.

Lusakowski, J.; Knap, W.; Dyakonova, N.; Varani, L.; Mateos, J.; Gonzalez, T.; Roelens, Y.; Bollaert, S.; Cappy, A. \& Karpierz, K. (2005). Terahertz emission by plasma waves in $60 \mathrm{~nm}$ gate high electron mobility transistors, J. Appl. Phys. Vol.97, No. 13 (February 2005) pp. 064307 1-3, ISSN 1077-3118

Maestrini, A.; Thomas, B.; Wang, H.; Jung, C.; Treuttel, J.; Jin, Y.; Chattopadhyay, G.; Mehdi, I. \& Beaudin, G. (2010). Schottky diode-based terahertz frequency multipliers and mixers , Comptes Rendus Physique, Vol.11, No.7, (2010). pp. 480-495, ISSN 1631-0705

Magno, R.; Champlain, J. G.; Newman, H. S.; Ancona, M. G.; Culbertson, J. C.; Bennett, B. R.; Boos, J. B. \& Park D., (2008). Antimonide-based diodes for terahertz mixers, Appl. Phys. Lett. Vol.92, No. 24, (June, 2008) pp. 243502 1-3, ISSN 1077-3118

Marandi, A.; Darcie, T. E.; and So, P. P. M. (2008). Design of a continuous-wave tunable terahertz source using waveguide-phase-matched GaAs, Optics Express, Vol.16, No.14, (June 2008) pp 10427-10433, ISSN 1094-4087

Matsuo, H. (2006). Future prospects of superconducting direct detectors in terahertz frequency range, Nuclear Instruments and Methods in Physics Research A, Vol.559, (January 2006), pp. 748-750, ISSN 0168-9002

McLaughlin, C. V.; Hayden, L. M.; Polishak, B.; Huang, S.; Luo, J. Kim, T. \& Jen, A. K. (2008). Wideband $15 \mathrm{THz}$ response using organic electro-optic polymer emitter-sensor pairs at telecommunication wavelengths, Appl. Phys. Lett. Vol.92, No.15, (April 2008), pp. 151107 1-3, ISSN 1077-3118

Meledin, D. V.; Marrone, D. P.; Tong, C. Y.; Gibson, .H.; Blundell, R.; Paine, S. N.; Papa, D. C.; Smith, M.; Hunter, T. R.; Battat, J.; Voronov, B. \& Gol'Tsman, G. A 1-THz superconducting hot-electron-bolometer receiver for astronomical observations, IEEE Trans. on Microwave Theory and Techniques, Vol.52, No. 10, (October 2004), pp. 2338-2343, ISSN 0018-9480

Mendis, R.; Astley, V.; Liu, J. \& Mittleman, D. M. (2009). Terahertz microfluidic sensor based on a parallel-plate waveguide resonant cavity, Appl. Phys. Lett. Vol.95, No.17, (October 2009), pp.171113, ISSN 1077-3118

Mittleman, D. (2003). in Sensing with Terahertz Radiation Springer, ISBN 3-540-43110-1, New York

Miyadera, T.; Kiwa, T.; Kawayama, I.; Murakami, Tonouchi, H. M. (2004). Ultrafast optical study of amorphous Ge thin films for superconductor/semiconductor hybrid devices, Physica C: Superconductivity, Vol.412, No.2 (July 2004) pp.1602-1606, ISSN 0921-4534

Molis G., Adomavicus R., Krotkus A., (2008) Temperature-dependent terahertz radiation from the surfaces of narrow-gap semiconductors illuminated by femtosecond laser pulses, Physica B, Vol.403 (2008) pp. 3786-3788.

Mueller E.R., (2003) Terahertz Radiation: Applications and Sources, The Industrial Physicist, (Aug-Sept. 2003), pp 27-29.

Nagel, M.; Först, M \& Kurz, H (2006). THz biosensing devices: fundamentals and technology, J. Phys.: Condens. Matter, Vol.18, No.18, (April 2006), pp. S601, ISSN 1361-648X

Nagel M, Richter F., Bolivar PH, \& Kurz H (2003), A functionalized THz sensor for marker free DNA analysis Phys. Med. Biol. Vol.48 (2003) pp.3625-36. ISSN: 0031-9155

Nikoghosyan, A.S. (2010). Laser Driven Terahertz Dielectric Wedge Antenna laced in Free Space or in Hollow Metallic Waveguide, 35th International Conference on Infrared 
Millimeter and Terahertz Waves (IRMMW-THz), (September 2010) pp. 1-2, ISBN 9781-4244-6655-9, Rome,

Otsuji, T., Tsuda, Y., Komori, T., El Fatimy, A., Suemitsu, T. (2009) Terahertz plasmonresonant microship emitters and their possible sensing and spectroscopic applications Proceedings of IEEE Sensors, art. no. 5398309, (2009) pp. 1991-1996.

Otsuji, T.; Karasawa, H.; Watanabe, T.; Suemitsu, T.; Suemitsu, M.; Sano, E. Knap, W.; Ryzhii, V. (2010). Emission of terahertz radiation from two-dimensional electron systems in semiconductor nano-heterostructures, Comptes Rendus Physique, Vol.11, No.7, (July 2010), pp. 421-432, ISSN 1631-0705

Peroz, C.; Degardin, A. F.; Villegier, J. C. \& Kreisler, A. (2007). Fabrication and Characterization of Ultrathin $\mathrm{PBCO} / \mathrm{YBCO} / \mathrm{PBCO}$ Constrictions for Hot Electron Bolometer THz Mixing Application, J. Appl. Supercond., IEEE Trans., Vol.17 , No. (July 2007), pp. 637-640, ISSN 1051-8223

Pilla, S (2007). Enhancing the photomixing efficiency of optoelectronic devices in the terahertz regime, Appl. Phys. Lett., Vol.90, No.16, (April 2007), pp. 161119-22, ISSN 1077-3118

Preu, S., Dhler, G.H., Malzer, S., Wang, L.J., Gossard, A.C. (2011) Tunable, continuous-wave Terahertz photomixer sources and applications Journal of Applied Physics Vol.109 No.6, (2011) art. no. 061301

Putz, P.; Jacobs, K.; Justen, M.; Schomaker, F.; Schultz, M.; Wulff, S. \& Honingh, C. E (2011). NbTiN Hot Electron Bolometer Waveguide Mixers on $\$\{r m \text { Si }\}_{-}\{3\}\{r m ~ N\}_{-}\{4\} \$$ Membranes at THz Frequencies, Appl. Superconductivity, IEEE Trans. Vol.21, (May 2011), pp. 636-639, ISSN 1051-8223

Radhanpura, K.; Hargreaves, S.; Lewis, R. A. \& Henini, M. (2009). The role of optical rectification in the generation of terahertz radiation from GaBiAs, Appl. Phys. Lett. Vol.94, No. 25, (June 2009), pp. 251115-1-3, ISSN 1077-3118

Radhanpura, K.; Hargreaves, S.; Lewis, R. A.; Sirbu, L. \& Tiginyanu, I. M. (2010), Heavy noble gas ( $\mathrm{Kr}, \mathrm{Xe})$ irradiated (111) InP nanoporous honeycomb membranes with enhanced ultrafast all-optical terahertz emission, Appl. Phys. Lett. Vol.97, No. 18, (November 2010), pp. 181921-1-3, ISSN 1077-3118

Rangan C. \& Bucksbaum P.H., Optimally shaped THz pulses for quantum algorithm on a Rydberg atom register, Physical Review A, Vol. 64 (2001) pp 37402- 37410.

Reklaitis A. , (2011) Crossover between surface field and photo-Dember effect induced terahertz emission, Journal of Applied Physics, Vol. 109, (2011) pp. 083108(5 pages)

Reimann K., (2007), Table-top sources of ultrashort THz pulses, Reports on Progress in Physics, Vol. 70 (2007) pp.1597.

Rau, C.; Torosyan, G.; Beigang, R. \& Nerkararyan, Kh., (2005). Prism coupled terahertz waveguide sensor, Appl. Phys. Lett. Vol.86, No.21, (2005), pp. 2111191-3, ISSN 1077-3118

Reid, I.; Cravetchi, R.; Fedosejevs, I. M.; Tiginyanu, L.; Sirbu, \& Robert W. Boyd, (2008). Enhanced nonlinear optical response of InP(100) membranes, Phys. Rev. B Vol.71, No. 8, (February 2005), pp. 081306, ISSN 1550-235x

Rinzan, M. B.; M. Perera, A. G. U.; Matsik, S. G.; Liu, H. C.; Wasilewski, Z. R. \& Buchanan, M. (2004). AlGaAs emitter/GaAs barrier terahertz detector with a $2.3 \mathrm{THz}$ threshold, Appl. Phys. Lett., Vol.86, No.7 (Februvary 2005), pp. 071112 1-3, ISSN 1077-3118

Roser H.P., Hubers H.W., Crowe T.W., \& Peatman W.C.B., Nanostructure GaAs Schottky diodes for far-infrared heterodyne receivers, IR Physics vol. 35 (1994) 451.

Roeser H.P., Haslam D.T., Hetfleisch F., Lopez J.S., vonSchoenermark M.F., Stepper M., Huber F.M., Nikoghosyan A.S., (2010) Electron transport in nanostructures: A key 
to high temperature superconductivity? Acta Astronautica, Vol. 67 (2010) pp. 546-552.

Ryzhii, V.; Satou, A.; Knap, W.; \& Shur, M. S. (2006). Plasma oscillations in high-electronmobility transistors with recessed gate, J. Appl. Phys. Vol.99, No.8, (May 2006), pp. 084507-1-5 ISSN 1089-7550

Sakai K., \& Tani M., (2005) Introduction to Terahertz pulses, Topics in Applied Physics, Vol. 97 (2005), pp. 1-30.

Schultz, J. \& Lichtenberger, A. (2007). Investigation of Novel Superconducting Hot Electron Bolometer Geometries Fabricated With Ultraviolet Lithography, Applied Superconductivity, IEEE Trans. Vol.17, (July 2007), No. 2, pp. 645-648, ISSN 1051-8223

Shchepetov, A.; Gardès, C.; Roelens, Y.; Cappy, A.; Bollaert, S.; Boubanga-Tombet, S.; Teppe, F.; Coquillat, D.; Nadar, S.; Dyakonova, N.; Videlier, H.; Knap, W.; Seliuta, D.; Vadoklis, R. and Valušis G. (2008). Oblique modes effect on terahertz plasma wave resonant detection in InGaAs/InAlAs multichannel transistors, Appl. Phys. Lett. Vol.92, No.24, (June 2008), pp. 242105-1-3, ISSN 1077-3118

Shibuya, T.; Suizu, K.; and Kawase, K.; (2010). Widely Tunable Monochromatic Cherenkov Phase-Matched Terahertz Wave Generation from Bulk Lithium Niobate, Appl. Phys. Express, Vol.3, (August 2010), pp. 082201-082204 ISSN 1882-0786

Sizov, F. \& Rogalski A. (2010). THz detectors, Progress in Quantum Electronics, Vol.34, No.5, (September 2010), pp. 278-347, ISSN 0079-6727

Stern, J. A.; Bumble, B.; Kawamura, J. \& Skalare, A. (2005). Fabrication of terahertz frequency phonon cooled HEB mixers, IEEE Trans. Appl. Superconductivity, Vol.15, No. (June 2005), pp. 499-502, ISSN 1051-8223

Suen, J. Y.; Li, W.; Taylor, Z. D. and Brown, E. R. (2010). Characterization and modeling of a terahertz photoconductive switch, Appl. Phys., Lett. Vol.96, No.14 (April 2010) pp. 141103-141106 ISSN 1077-3118

Suzuki, T.; Yasui, T.; Fujishima, H.; Nozokido, T.; Araki, M.; Boric-Lubecke, O.; Lubecke, V.M.; Warashina, H. \& Mizuno, K. (1999). Microwave Theory and Techniques, IEEE Trans., Vol.47 No.9, (September 1999), pp. 1649 - 1655, ISSN 0018-9480

Tochitsky S. Ya., Ralph J. E., Sung C., \& Joshi C.(2005), Generation of megawatt-power terahertz pulses by noncollinear difference-frequency mixing in GaAs, Journal of Applied Physics, Vol. 98, No.2 (2005) 026101-1-3.

Tonouchi, M. (2007). Cutting-edge terahertz technology, Nature Photoic., Vol.1, (February 2007), pp. 97-105, ISSN 1749-4885

Tredicucci, A.; Kohler, R.; Mahler, L.; Beere, H. E.; Linfield, E. H. \& Ritchie, D.A. (2005). Terahertz quantum cascade lasers-first demonstration and novel concepts, Semicond. Sci. Technol. Vol.20, No.7, (June 2005) pp. S222-S227, ISSN 1361-6641

Tsaur G. \& Wang, J. (2009). Relativistic optical rectification driven by a high-intensity pulsed Gaussian beam, Phys. Rev. A, Vol.80, No.2 (August 2009) pp. 023802-1-11 ISSN 1094-1622

Turchinovich D \& Dijkhuis J.I. (2007) Performance of combined $<100>-<110>$ ZnTe crystals in an amplified $\mathrm{THz}$ time-domain spectrometer, Optics Communications Vol. 270 (2007) pp. 96-99

Ueda T \& Komiyama S (2010) Novel Ultra-Sensitive Detectors in the 10-50 mm wave length range, Sensors, Vol. 10, (2010) 8411-8423 ISSN 1424-8220

Urbanowicz, A.; Krotkus, A.; Adomavičius, R. \& Malevich, V.L. (May 2007). Terahertz emission from femtosecond laser excited Ge surfaces due to the electrical field- 
induced optical rectification, Physica B: Condensed Matter, Vol.398, No. 98 (May 2007), pp. 98-101, ISSN 0921-4526

Valavanis, A.; Dinh, T. V.; Lever, L. J. M.; Ikonić, Z. and Kelsall, R. W. (2011). Material configurations for n-type silicon-based terahertz quantum cascade lasers Phys. Rev. B, Vol.83, No.19, (May 2011) pp. 195321-195329 ISSN 1550-235x

Vidal, S.; Degert, J.; Tondusson, M.; Oberlé, J. \& Freysz, E. (2011). Impact of dispersion, free carriers, and two-photon absorption on the generation of intense terahertz pulses in ZnTe crystals, Appl. Phys. Lett. Vol.98, No. 19, (May 2011), pp. 191103-1-3, ISSN 1077-3118

Videlier, H., Nadar, S., Dyakonova, N., Sakowicz, M., Trinh Van Dam, T., Teppe, F., Coquillat, D.,\&Lyonnet, J., ( 2009), Silicon MOSFETs as room temperature terahertz detectors, Journal of Physics: Conference Series Vol. 193, (2009) art. no. 012095.

Williams G.P., High-power terahertz synchrotron sources, Philosophical Transactions of the Royal Society London A vol. 362 (2004), pp. 403-414. ISSN: 1471-2962.

Xiao-li, Z. \& Jiu-sheng, L. (2011). Diagnostic techniques of talc powder in flour based on the THz spectroscopy, J. Phys.: Conf. Ser. Vol.276, No.1, (March 2011) pp.012234 ISSN 1742-6596

Yang, X.; Qi, S.; Zhang, C.; Chen, K.; Liang, X.; Yang, G.; Xu, T.; Han, Y. and Tian, J. (2011), The study of self-diffraction of mercury dithizonate in polymer film, Optics Commun. Vol.256, No. 4, (July 2011) pp. 414-421, ISSN 0030-4018

Yasuda, H.; Kubis, T.; Vogl, P.; Sekine, N.; Hosako, I. \& K. Hirakawa, (2009). Nonequilibrium Green's function calculation for four-level scheme terahertz quantum cascade lasers, Appl. Phys. Lett. Vol.94, No.15, (April 2009), pp. 151109 1-3, ISSN 1077-3118

Yasui, T.; Nishimura, A.; Suzuki, T.; Nakayama, K. \& Okajima S. (2006). Detection system operating at up to $7 \mathrm{THz}$ using quasioptics and Schottky barrier diodes, Rev. Sci. Instrum. Vol.77, No.6, (2006) pp-066102 1-3, ISSN 1089-7623

Yi, M., Lee, K., Lim, J., Hong, Y., Jho, Y.-D., \& Ahn J. (2010) Terahertz Waves Emitted from an Optical Fiber Optics Express vol. 18 No. 13, (2010) pp. 13693-13699.

Yu, N.E.; Lee, K.S.; Ko, D.-K. C. 1.; Kang.; Takekawa, S. 1. \& Kitamura, K. (2011). Temperature dependent narrow-band terahertz pulse generation in periodically poled crystals via difference frequency generation, Optics Commun., Vol.284 No.5, (November 2011), pp. 1395-1400, ISSN 0030-4018

Zhang, L.; Zhong, H.; Deng, C.; Zhang, C. \& Zhao, Y. (2008). Terahertz wave reference-free phase imaging for identification of explosives, Appl. Phys. Lett. Vol.92, No.9, (March 2008), pp. 091117, ISSN 1077-3118

Zheng, X., McLaughlin, C.V., Cunningham, P., Hayden, L.M. (2007), Organic broadband terahertz sources and sensors, Journal of Nanoelectronics and Optoelectronics Vol. 2 No.1 (2007), pp. 58-76.

Zheng, X., McLaughlin, C.V., Cunningham, P., Hayden, L.M. (2007), Organic broadband terahertz sources and sensors, Journal of Nanoelectronics and Optoelectronics Vol. 2 No.1 (2007), pp. 58-76.

Zmuidzinas J., Richards P.L., (2004), Superconducting detectors and mixers for Millimeter and sub-millimeter Astrophysics, Proceedings of IEEE, vol.192, No.10, (October 2004) pp 1597-1616. 


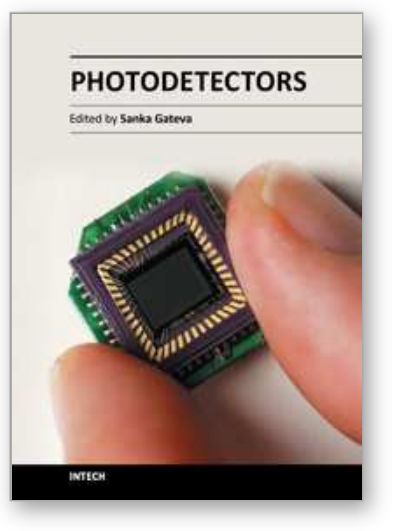

\author{
Photodetectors \\ Edited by Dr. Sanka Gateva
}

ISBN 978-953-51-0358-5

Hard cover, 460 pages

Publisher InTech

Published online 23, March, 2012

Published in print edition March, 2012

In this book some recent advances in development of photodetectors and photodetection systems for specific applications are included. In the first section of the book nine different types of photodetectors and their characteristics are presented. Next, some theoretical aspects and simulations are discussed. The last eight chapters are devoted to the development of photodetection systems for imaging, particle size analysis, transfers of time, measurement of vibrations, magnetic field, polarization of light, and particle energy. The book is addressed to students, engineers, and researchers working in the field of photonics and advanced technologies.

\title{
How to reference
}

In order to correctly reference this scholarly work, feel free to copy and paste the following:

M. Ghanashyam Krishna, Sachin D. Kshirsagar and Surya P. Tewari (2012). Terahertz Emitters, Detectors and Sensors: Current Status and Future Prospects, Photodetectors, Dr. Sanka Gateva (Ed.), ISBN: 978-953-510358-5, InTech, Available from: http://www.intechopen.com/books/photodetectors/thz-detectors-emitters-andsensors-current-status-and-future-prospects

\section{INTECH}

open science | open minds

\section{InTech Europe}

University Campus STeP Ri Slavka Krautzeka 83/A 51000 Rijeka, Croatia Phone: +385 (51) 770447

Fax: +385 (51) 686166 www.intechopen.com

\section{InTech China}

Unit 405, Office Block, Hotel Equatorial Shanghai

No.65, Yan An Road (West), Shanghai, 200040, China 中国上海市延安西路65号上海国际贵都大饭店办公楼 405 单元 Phone: +86-21-62489820

Fax: +86-21-62489821 
(C) 2012 The Author(s). Licensee IntechOpen. This is an open access article distributed under the terms of the Creative Commons Attribution 3.0 License, which permits unrestricted use, distribution, and reproduction in any medium, provided the original work is properly cited. 